\title{
A novel bioinformatics pipeline to discover genes related to arbuscular mycorrhizal symbiosis based on their evolutionary conservation pattern among higher plants
}

Patrick Favre ${ }^{1,3,5}$, Laure Bapaume ${ }^{1}$, Eligio Bossolini ${ }^{1,6}$, Mauro Delorenzi ${ }^{2,4,5}$, Laurent Falquet ${ }^{1,3}$ and Didier Reinhardt ${ }^{1 *}$

\begin{abstract}
Background: Genes involved in arbuscular mycorrhizal (AM) symbiosis have been identified primarily by mutant screens, followed by identification of the mutated genes (forward genetics). In addition, a number of AM-related genes has been identified by their AM-related expression patterns, and their function has subsequently been elucidated by knock-down or knock-out approaches (reverse genetics). However, genes that are members of functionally redundant gene families, or genes that have a vital function and therefore result in lethal mutant phenotypes, are difficult to identify. If such genes are constitutively expressed and therefore escape differential expression analyses, they remain elusive. The goal of this study was to systematically search for AM-related genes with a bioinformatics strategy that is insensitive to these problems. The central element of our approach is based on the fact that many AM-related genes are conserved only among AM-competent species.
\end{abstract}

Results: Our approach involves genome-wide comparisons at the proteome level of AM-competent host species with non-mycorrhizal species. Using a clustering method we first established orthologous/paralogous relationships and subsequently identified protein clusters that contain members only of the AM-competent species. Proteins of these clusters were then analyzed in an extended set of 16 plant species and ranked based on their relatedness among AM-competent monocot and dicot species, relative to non-mycorrhizal species. In addition, we combined the information on the protein-coding sequence with gene expression data and with promoter analysis. As a result we present a list of yet uncharacterized proteins that show a strongly AM-related pattern of sequence conservation, indicating that the respective genes may have been under selection for a function in AM. Among the top candidates are three genes that encode a small family of similar receptor-like kinases that are related to the S-locus receptor kinases involved in sporophytic self-incompatibility.

Conclusions: We present a new systematic strategy of gene discovery based on conservation of the protein-coding sequence that complements classical forward and reverse genetics. This strategy can be applied to diverse other biological phenomena if species with established genome sequences fall into distinguished groups that differ in a defined functional trait of interest.

Keywords: Arbuscular mycorrhiza, Symbiosis, Symbiosis signaling, Common SYM gene, Conservation, Gene clustering, Proteome analysis, Bioinformatics

\footnotetext{
* Correspondence: didier.reinhardt@unifr.ch

'Department of Biology, University of Fribourg, Fribourg, Switzerland

Full list of author information is available at the end of the article
} 


\section{Background}

Most land plants engage in symbiotic associations with fungi (Glomeromycota) that colonize their roots and provide them with phosphate and other mineral nutrients [1]. This association, referred to as arbuscular mycorrhiza (AM), is found in most major taxa of land plants [2], and is thought to have emerged monophyletically in an early progenitor of the vascular plants [3]. The strongest argument for this assumption is the fact that mycorrhizal development requires a conserved signalling pathway that consists of approximately 10 genes that encode receptor components such as SYMRK, and signalling intermediates such as CCaMK [4]. The genes involved in this pathway are conserved between monocots and dicots, and occur also in lycopods and mosses [5,6], suggesting that the origin of AM dates back to the early vascular plants at the time when land became colonized [3]. This assumption is consistent with the fossil record, which provides evidence for AM-like associations in the sediments of the Rhynie chert that is estimated to originate from the Ordovician period around $450 \mathrm{My}$ ago [7]. More than $350 \mathrm{My}$ after the evolution of AM, a subsequent event in a small subset of the dicots (Fabales, Fagales, Cucurbitales, Rosales), allowed for the emergence of a new form of symbiosis, root nodule symbiosis (RNS) with rhizobacteria [8-10]. Interestingly, RNS, as well as the actinorrhizal symbiosis with cyanobacterial endosymbionts [11,12], involve the same signalling pathway as AM, which therefore is referred to as common symbiosis signalling pathway (common SYM pathway) $[4,13]$. A central element of the common SYM pathway is calcium spiking, a rythmic change in perinuclear calcium concentration, which is perceived and transmitted by calcium and calmodulin-dependent protein kinase (CCaMK) to induce symbiotic gene expression $[14,15]$.

AM is formed by more than $80 \%$ of the vascular plants [1], indicating that this association provides a significant selective advantage over non-mycorrhizal plants. However, some plant taxa do not form AM, among them the Brassicaceae with the best-characterized model plant species Arabidopsis thaliana, and the Chenopodiaceae with the economically important crop species Beta vulgaris (sugar beet). AM-related genes are often conserved among AM-competent plant species, while they are less conserved or even missing in non-mycorrhizal species. This phenomenon has been described for VAPYRIN (VPY), which is essential for infection and development of the fungal feeding structures, the arbuscules, in Petunia hybrida as well as in Medicago truncatula [16,17]. VPY is entirely missing from non-mycorrhizal plant species [16-18], and the same is true for numerous genes that are expressed specifically in AM $[19,20]$. Such a pattern of conservation was also observed in AM-related genes that are members of large ubiquitous gene families such as the
ABC transporters STUNTED ARBUSCULE (STR) and STR2, or the GRAS-type transcription factor REQUIRED FOR ARBUSCULAR MYCORRHIZA1 (RAM1), which both belong to subfamilies which are restricted to AM-competent plant species [21,22]. Similarly, several components of the common SYM pathway are missing from the non-mycorrhizal model species Arabidopsis thaliana [6], whereas they are conserved among AM-competent dicots and monocots.

Traditionally, AM-related genes have been identified either by mutant screenings followed by characterization of the mutated gene (forward genetics), or by transcript profiling, followed by mutational analysis of AM-induced genes (reverse genetics). Considering the increasing number of sequenced plant genomes, the loss of AM-related genes from the genomes of non-mycorrhizal species could serve as a criterion to detect new AM-related genes by comparative genomics. This third way of gene discovery could potentially identify AM-related genes that have escaped characterization via traditional genetic approaches because of functional redundancy, lethal phenotypes, or constitutive gene expression. Conceptually, such an approach represents a substractive procedure where the proteomes of non-mycorrhizal plants such as $A$. thaliana are substracted from a panel of proteomes of AM-competent species to result in a set of proteins that are consistently conserved among AM-competent plants and absent from non-mycorrhizal reference species.

Here, we describe a novel approach to identify new AM-related genes based on genome substraction. The approach consists of a multistep procedure that uses protein sequence conservation and gene expression as criteria for the enrichment of potential symbiosis-related genes. We have compared a set of six AM-competent angiosperm species (Solanum lycopersicum, Solanum tuberosum, Vitis vinifera, Medicago truncatula, Glycine max, Populus trichocarpa), and three non-AM species (Arabidopsis thaliana, Arabidopsis lyrata, and Brassica rapa) with an initial clustering approach that resulted in a set of potential candidate proteins comprising approximately $10 \%$ of the entire proteome. At a second step, a clustering of these genes based on gene expression patterns in Medicago truncatula provided a set of conserved genes that are induced in AM. Finally, to focus on conserved constitutively expressed genes (such as the common SYM genes), a proteome blast of the conserved genes to an extended panel of proteomes (including monocots) allowed to perform quantitative statistics on the E-values, hence providing a set of proteins that are significantly more conserved among AM-competent plant species than towards the nonmycorrhizal Brassicaceae. This strategy was validated with a number of known AM-related genes that passed our selection scheme. The resulting list of predicted AM-related 
proteins will be functionally tested by reverse genetic approaches.

\section{Results}

Patterns of sequence conservation in AM-related genes In order to explore the potential for differential conservation of AM-related genes, we established the phylogeny of two central AM-related proteins, the GRAS-type transcription factor REQUIRED FOR ARBUSCULAR MYCORRHIZA1 (RAM1), which is an essential regulator of AM symbiosis [21], and PHOSPHATE TRANSPORTER4 (PT4), which is required for symbiotic phosphate transfer and arbuscule functioning [23,24] (Additional file 1: File S1). A phylogenetic tree of RAM1 shows a clear bisection between mycorrhizal plants (group A and B), and non-mycorrhizal plants (group C) (Figure 1a). Notably, the sequences of AM-competent dicots and monocots (groups A and B) grouped significantly closer together than the dicots among each other (groups A and C). A similar pattern was observed in a phylogenetic tree of PT4 and its closest homologues in various monocot and dicot species (Figure 1b). As with RAM1, the homologues from AM-competent dicots (group A) and monocots (group B) grouped more closely together than the homologues of the phylogenetically related groups of the AM-competent dicots (group A) and the non-mycorrhizal dicots (group C).

A contrasting pattern was observed when two housekeeping genes were analysed, that encode cyclin D6 and chloroplast ribosomal protein L5 (Figure 1c and d). These proteins showed a pattern of conservation that reflects the closer relationship among the dicots, relative to the monocots, which clustered separately from all the dicot species (Figure 1c and d). Taken together, this evidence suggests that PT4 and RAM1, and perhaps other AM-related genes, are under diversifying selection in AM-competent species. Hence, AM-related genes could potentially be identified based on the relative conservation pattern of their encoded proteins.

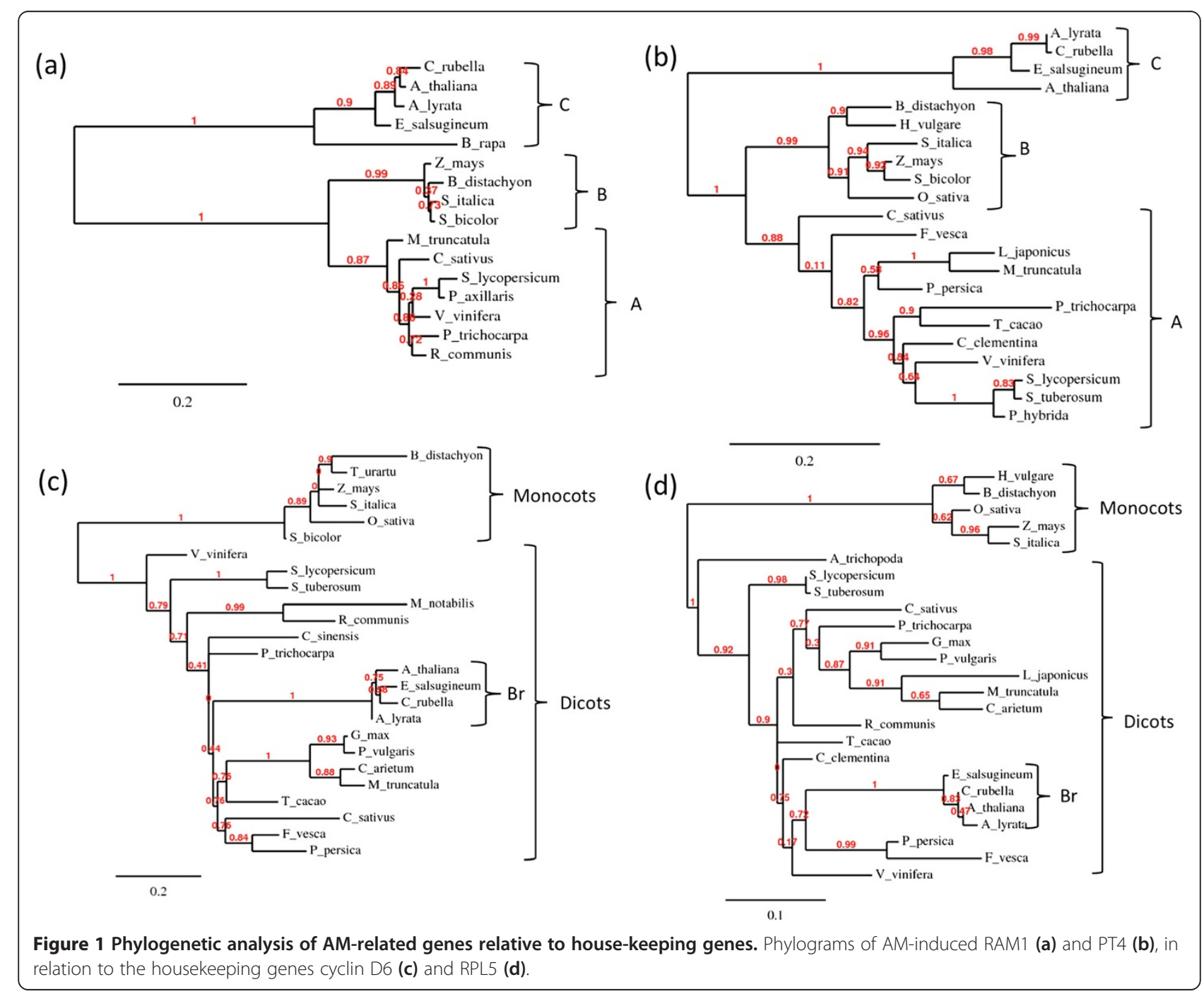


Hierarchical clustering to identify protein phylogeny In order to identify AM-related genes in a systematic way, we first applied the clustering software Hieranoid [25] to the proteome sequences of six AM-competent species, namely Medicago truncatula (Mtr), Glycine max (Gma), Vitis vinifera (Vvi), Solanum lycopersicum (Sly), Solanum tuberosum (Stu) and Populus trichocarpa (Ptr), and three non-mycorrhizal species, namely Arabidopsis thaliana (Ath), Arabidopsis lyrata (Aly), and Brassica rapa (Bra). Pairwise clustering proceeded based on a conceptual phylogenetic tree of the involved plant species (Additional file 2: Figure S1; Additional file 3: File S2, script P0_HieraProcedure.txt). Figure 2 describes the work-flow of our strategy. Briefly, the proteomes of 9 species were used for clustering. The resulting trees of orthologous/paralogous proteins were filtered to yield lists of protein clusters that satisfied certain defined criteria referred to as Task3, Task4, Task9 (see next section). These gene lists were further processed to isolate AM-related genes based on gene expression, on conservation of the protein-coding region, and on the promoter sequence, as discussed in detail in the following sections. Scripts involved in the different processes (P0, P1, P2, P3, and P4 in Figure 2) are provided in Additional file 3: File S2.

After an initial round of clustering, we noticed that, unexpectedly, the known AM-related VAPYRIN protein was not found among the trees generated by Hieranoid, although it is believed to generally occur in all AMcompetent plants $[17,26]$, whereas it is absent from the Brassicaceae [16,18]. Closer inspection of the Mtr proteome

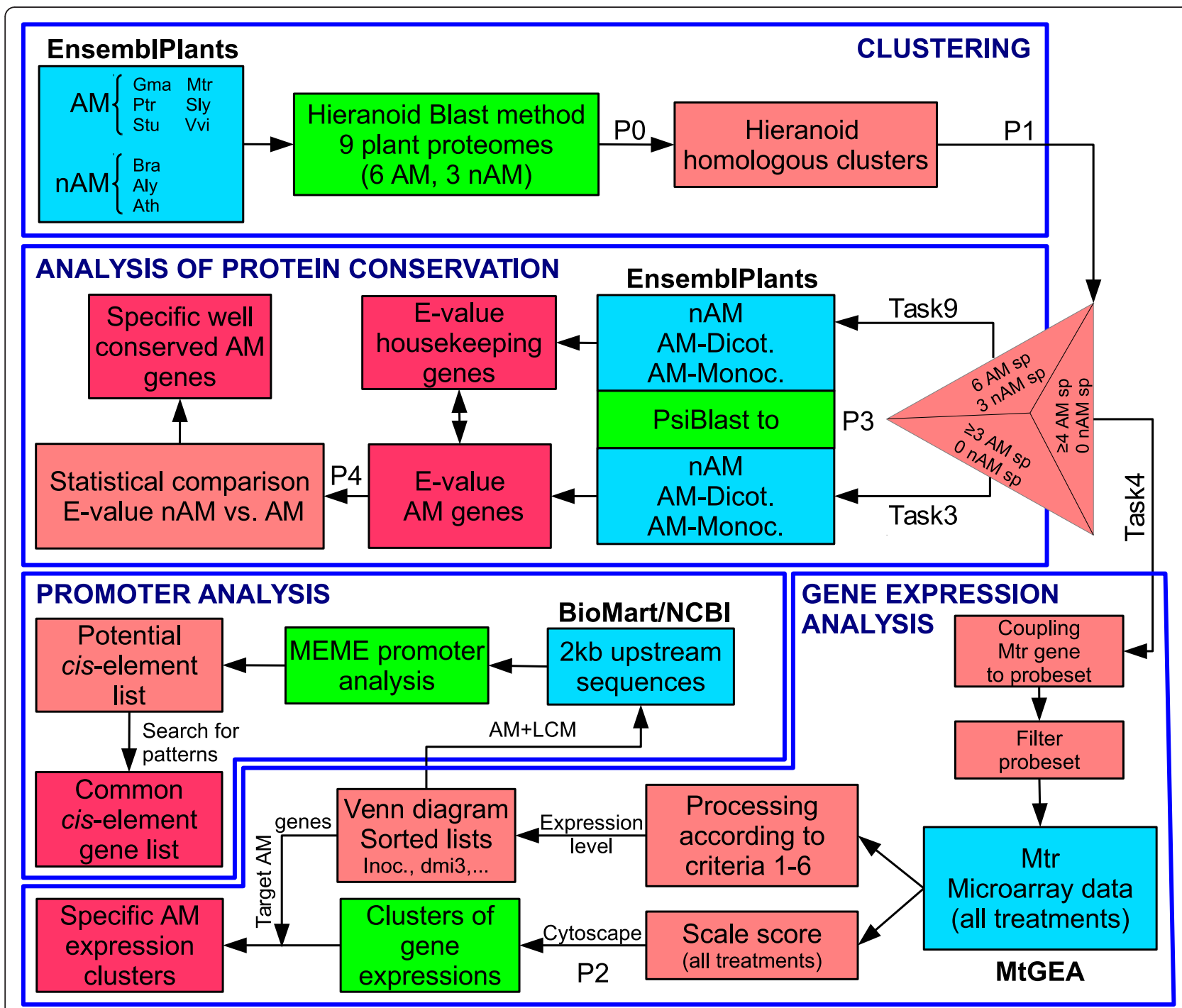

Figure 2 Strategy used to identify AM-related genes based on sequence conservation. The flow chart reflects the stepwise identification of potential AM-related proteins based on their pattern of sequence conservation at the protein level, the pattern of gene expression, and predicted regulatory elements in their promoters. Sp: Plant species. P0-P4 correspond to protocols, files, or scripts provided in supplementary materials (Additional file 4: File S3). Blue boxes: Databases; green boxes: Tools and processes; pink boxes: intermediate outputs; red boxes: final outputs. 
from ENSEMBL revealed that VAPYRIN, as well as the two AM-related genes CASTOR and PT4 were missing from the $M$. truncatula proteome (database: Ensembl Plants release 20). However, they could be identified in the UniProtKB database [27] and were added to the Mtr proteome (VAPYRIN: Mtr_D3J162; CASTOR: Mtr_D6C5X5; PT4: Mtr_AAM76743.1), and Hieranoid was restarted. Final clustering from the proteomes of the 9 plant species gave rise to a total of 28'528 clusters of orthologous and/or paralogous proteins (Figure 2, Additional file 4: File S3).

\section{Selecting clusters with potential AM-related proteins}

In order to select among all the clusters generated by Hieranoid those that showed the conservation pattern known for AM-related proteins (Figure 1a,b), we performed in silico substraction by selecting protein clusters that contained at least one protein of each of the 6 AM-competent species, but none of the nonmycorrhizal species Ath, Aly, and Bra (Task6). In order to account for cases where an individual protein may be missing because of an incomplete proteome (as observed in M. truncatula for VAPYRIN, CASTOR and PT4; see above), we also performed a more permissive search for clusters that contained proteins of at least 5 of the 6 AM-competent species, but none from the three non-mycorrhizal species (Task5), and we also carried out the corresponding subtractions with Task4 and Task3 (Figure 2, P1; see P1_hieranoid_output_treatments.sh in Additional file 3: File S2).

The numbers of clusters passing through these filters are listed in Table 1. In all filtering trials, M. truncatula showed considerably lower numbers than the other species, indicating that its proteome is less complete than those of the other AM-competent species (Table 1). Hence, to account for the incompleteness of the $M$. truncatula proteome, and for other potentially missing proteins, we selected the clusters identified by Task 4 for further analysis.

In order to assess the efficiency of Hieranoid clustering and subsequent filtering, we tested whether known AM-related genes passed the selection process. We assembled a list of 23 proteins with known function and/or expression pattern in AM (Additional file 5: Table S1, Additional file 6: File S4). They are known either as components of the common SYM signalling pathway (gene 1-9), as receptor of nod factor and potentially myc factor (NFP), as genes required specifically in AM development (RAM1, RAM2, STR), or as genes specifically induced in mycorrhizal roots (genes 14-19). An additional set of proteins served as negative controls that were not expected to pass the filter, either because they function as housekeeping genes and are therefore ubiquitous (PT1, PT6), or because they are primarily involved in nodulation but not AM (NODULATION SIGNALING PATHWAY1; NSP1).

As expected, most (5/8) of the components of the SYM signalling pathway passed the filter applied by Task4 (Additional file 5: Table S1), with the exception of DMI1/POLLUX and the nucleoporins NUP133, and NENA, which were known before to share close homologues with $A$. thaliana [28-30]. NUP85 was lost during the Hieranoid clustering and therefore cannot be used in this context. Furthermore, the LysM-type receptor kinase, as well as RAM1 and RAM2 were retained, whereas the ABC transporter STR was excluded. Genes that are induced during AM, or expressed exclusively in mycorrhizal roots, were also retained through filtering, with the exception of subtilase and PR10, with the latter being represented by a slightly more distant relative (MTR_2g035150 in Additional file 5: Table S1). Importantly, the negative controls (proteins encoded by constitutively expressed genes or by nodulation-specific

Table 1 Number of protein clusters from hierarchical clustering after filtering

\begin{tabular}{|c|c|c|c|c|}
\hline & Task3 & Task4 & Task5 & Task6 \\
\hline Number of clusters & 4438 & 2327 & 1184 & 479 \\
\hline Number of clusters with Sly & $3238(73 \%)$ & 1986 (85\%) & $1150(97 \%)$ & 479 (100\%) \\
\hline Number of clusters with Stu & $3205(72 \%)$ & $1959(84 \%)$ & 1137 (96\%) & 479 (100\%) \\
\hline Number of clusters with Vvi & $3138(71 \%)$ & 1967 (85\%) & 1057 (89\%) & $479(100 \%)$ \\
\hline Number of clusters with Gma & $2971(67 \%)$ & $1934(83 \%)$ & 1059 (89\%) & 479 (100\%) \\
\hline Number of clusters with Ptr & $2703(61 \%)$ & $1763(76 \%)$ & $1029(87 \%)$ & 479 (100\%) \\
\hline Number of clusters with Mtr & $2049(46 \%)$ & $1362(59 \%)$ & $867(73 \%)$ & 479 (100\%) \\
\hline Number of Mtr genes & 3203 & 2117 & 1338 & 717 \\
\hline Number of Mtr genes with at least one MtGEA probeset & 2373 & 1618 & 1028 & 563 \\
\hline Number of clusters with at least one MtGEA probeset & 1637 & 1109 & 708 & 392 \\
\hline
\end{tabular}

Among the total of $28^{\prime} 528$ clusters obtained from Hieranoid, those were selected that did not have a member from the Brassicaceae ( $A$. thaliana, $A$. lyrata, $B$. rapa), but had at least one hit from at least 3,4,5, or 6 of the AM-competent species, respectively. These are referred to as Task3, Task4, Task5 and Task6, respectively. In addition, the occurrence of the $6 \mathrm{AM}$-competent species in the respective clusters is indicated. Information of the relative representation of genes from M. truncatula (Mtr) in the Medicago gene Atlas is provided as well. 
genes) were eliminated by filtering (Additional file 5: Table S1). These results show that our clustering and filtering procedure has the potential to identify AM-related genes based on conservation of the protein sequence. The fact that STR was removed despite its conservation pattern that would be expected to allow it to pass through our filtering approach [22], can be explained with the fact that it is part of a large gene family (ABC transporters).

\section{Identifying AM-related proteins by gene expression pattern}

AM-related genes can potentially be identified based on their induction during AM development. Genome-wide analysis of AM-related gene expression has been performed in a number of plant species including M. truncatula, $L$. japonicus, S. lycopersicum (tomato), Oryza sativa (rice), and Petunia hybrida [19,31-35]. A large set of transcriptomic data is available online for the model legume $M$. truncatula (Medicago GeneAtlas; http://mtgea.noble.org/v3). We used this resource to identify among the protein clusters resulting from Task4 those that are induced at the transcriptional level during AM. We first extracted for all the trees retained by Task4 (2327 clusters) those that had a member from $M$. truncatula (1362 clusters). These clusters represented a total of 2117 genes of $M$. truncatula, of which 1618 genes had at least one Affymetrix probe set in the M. truncatula Gene Atlas (MtGEA). After removal of unreliable probesets (see Methods), the expression data for $1526 \mathrm{M}$. truncatula genes representing 1054 protein clusters were used for further analysis.

Expression data are available from various conditions including mycorrhizal roots with Rhizophagus irregularis and with Glomus mosseae, and laser-microdissected root cortex cells with arbuscules from $R$. irregularis. In addition, expression data of inoculated roots of the doesn't make infection3 (dmi3) mutant [36,37], roots treated with myc factor (mycLCO) for $6 \mathrm{~h}$ and $24 \mathrm{~h}$, and roots treated with low phosphate levels are available. The values of gene expression under these treatments and of the corresponding control treatments, were extracted from the Medicago Gene Atlas to calculate induction ratios according to 6 criteria (Table 2). In order to focus on proteins that are induced robustly in mycorrhizal roots, an induction threshold of 3-fold was applied for further filtering of candidate genes. A complete list of genes identified by Task 4 with the corresponding expression ratios according to criteria 1-6 is provided in Additional file 7: Table S2.

Combinatorial analysis revealed that 65 genes were commonly induced in mycorrhizal roots and in microdissected arbusculated cells, whereas 26 genes were induced only in mycorrhizal roots, and 173 were induced only in microdissected arbusculated cells, respectively (Figure 3, Additional file 8: Table S3). Only 7 genes were induced by mycLCO after $6 \mathrm{~h}$ of treatment, while none of them
Table 2 Criteria used for gene expression filtering of M. truncatula genes identified in Task4

\begin{tabular}{|c|c|c|c|}
\hline & Name (Figure 3) & & Name of treatment in MtGEA \\
\hline \multirow[t]{2}{*}{ Criterion 1} & LCM & test & Root LCM arbuscular \\
\hline & & cont & Root LCM cortical \\
\hline \multirow[t]{3}{*}{ Criterion 2} & AM & test* & $\begin{array}{l}\text { Root (28dpi) Myc (G. intraradices) } \\
6 \text { wk } 20 \text { uM P }\end{array}$ \\
\hline & & & $\begin{array}{l}\text { Root (28dpi) Myc (G. mosseae) } \\
6 \text { wk } 20 \text { uM P }\end{array}$ \\
\hline & & cont & Root non-Myc (control) 6wk 20 uM P \\
\hline \multirow[t]{2}{*}{ Criterion 3} & dmi3 & test & $\begin{array}{l}\text { Root DMI3 inoculated with Gigaspora } \\
\text { (early contact) }\end{array}$ \\
\hline & & cont & Root DMI3 control \\
\hline \multirow[t]{2}{*}{ Criterion 4} & MF_6 h & test & Root WT nsMyc-LCOs $6 \mathrm{~h}$ \\
\hline & & cont & Root WT MF control $6 \mathrm{~h}$ \\
\hline \multirow[t]{2}{*}{ Criterion 5} & MF_24 h & test & Root WT nsMyc-LCOs $24 \mathrm{~h}$ \\
\hline & & cont & Root WT MF control $24 \mathrm{~h}$ \\
\hline \multirow[t]{2}{*}{ Criterion 6} & P-repressed & test & Root non-Myc (control) 6wk 20 uM P \\
\hline & & cont & Root non-Myc $6 \mathrm{wk} 2 \mathrm{mM} P$ \\
\hline
\end{tabular}

These 6 criteria were applied to the 1526 genes of Task4 (Additional file 7: Table S2) for which a reliabel probe set was available in the $M$. truncatula Gene Atlas (http://mtgea.noble.org/v3). * Expression values were averaged for the two samples inoculated by $G$. intraradices and $G$. mosseae, respectively.

remained induced after $24 \mathrm{~h}$ of treatment (Figure 3). Interestingly, 10 genes were induced in the dmi3 mutant which is defective for CCaMK, indicating that their expression is regulated independently of the common SYM pathway. Three of these genes were also induced in AM roots, in microdissected arbusculated cells, and in P-starved roots (Table 1, criterion 6) (Figure 3). It will be interesting to explore how these genes are induced in the absence of DMI3.

While gene expression patterns can be identified based on defined conditions (e.g. criteria 1-6 in this study), global expression analysis by clustering can identify groups of genes with similar expression patterns over a large set of expression data like the 254 different treatments and conditions covered by the Medicago Gene Atlas (http://mtgea.noble.org/v3). This approach can identify groups of genes that are co-regulated and therefore might be functionally related. On the other hand, this approach can lead to the discovery of common regulatory elements in promoters, which are the reason for coregulation (see below). Hence, we decided to use pairwise average linkage and Pearson correlation in order to identify genes with shared expression pattern (Figure 2, P2; see P2_task4cytoallr.cys in Additional file 3: File S2). All proteins identified by Task 4 were correlated based on their standardized gene expression score, i.e. the ratios between the individual expression levels divided by the average of all expression levels as a relative indicator of expression (see Methods). Particular attention was paid to 


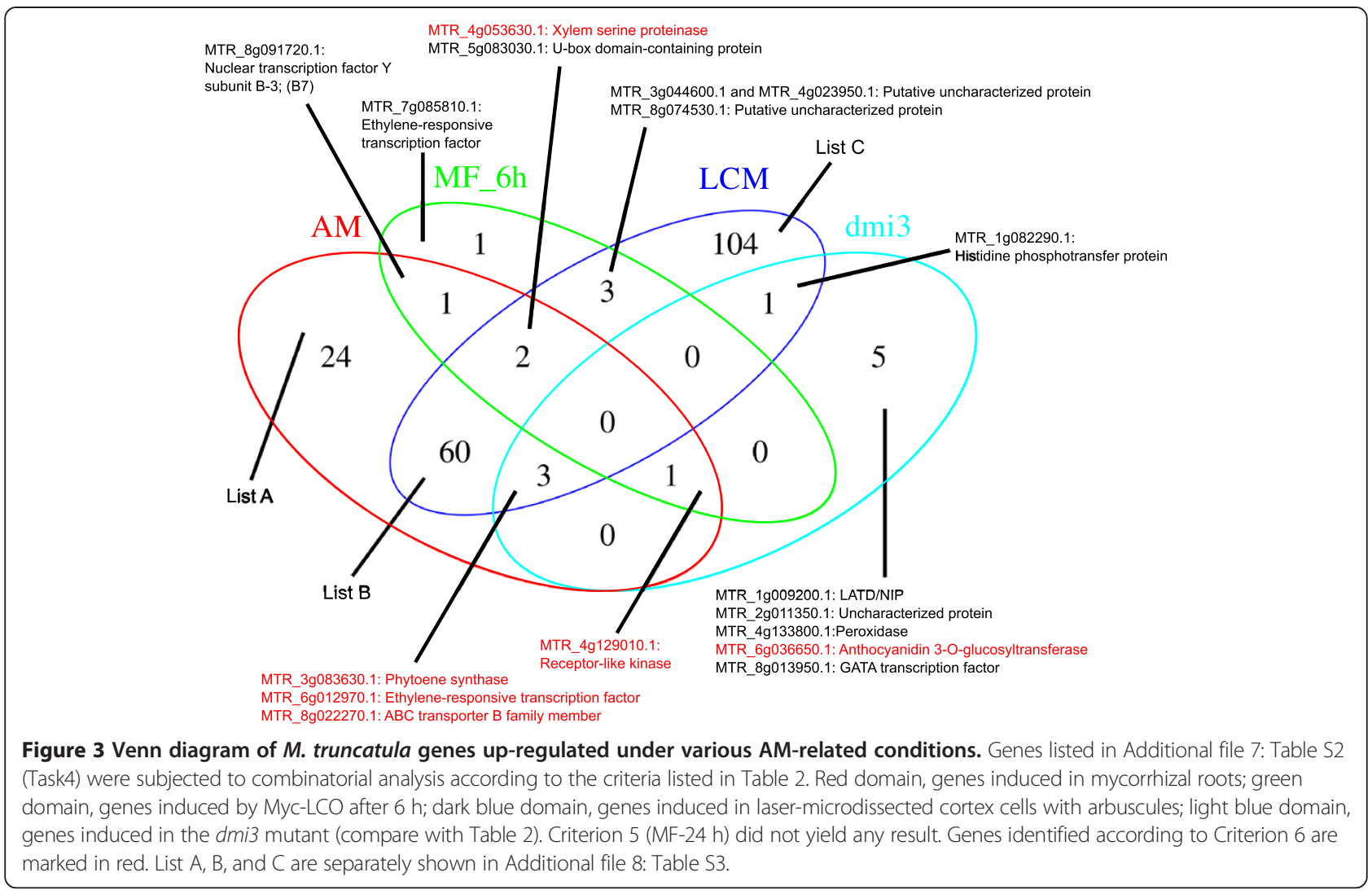

groups of genes that comprised AM-induced genes. One conspicuous cluster of 51 genes with a significantly correlated expression pattern turned out to be highly specific for AM, resulting in apparent vertical red stripes in the visual representation of the cluster (Additional file 9: Figure S2 and Figure 4). A further relevant group comprised genes that are induced commonly in AM and in RNS (Additional file 10: Figure S3 and Additional file 11: Figure S5). These genes may encode proteins that play a general role in symbiotic interactions. Interestingly, this cluster consisted primarily of chitinases, cysteine proteases, a glucanase and several ripening-related proteins (Figure 5).

\section{Analysis of relative conservation of proteins among angiosperms}

Many genes with a role in AM are induced during the interaction. This includes nutrient transporters such as AM-related phosphate transporters [23,38-40] (see also above), and the ammonium transporter AMT2 [41], as well as regulatory components such as the transcription factor RAM1 [21]. In contrast, the genes involved in the early steps of the interaction are constitutively expressed. For example, the expression of the nod factor receptors (NFRs) and of the common SYM genes is not significantly altered during AM development in petunia [19], consistent with their early function in symbiont recognition and signalling.

In order to identify constitutively expressed genes with a potential role in AM development, we decided to compare the relative sequence conservation of proteins in the context of the three AM-relevant plant groups: AM-competent dicots (group A), AM-competent monocots (group B), and non-mycorrhizal dicots (group C) (compare with Figure 1a,b). In order to avoid to miss relevant genes due to proteome incompleteness, we chose the relatively permissive Task3 for this approach (clusters with at least 3 homologues of the 6 AM-competent species but none of the non-mycorrhizal species). Firstly, multiple sequence alignment (MSA) from the sequences represented in the individual clusters retained by Task 3 were calculated using MAFFT [42], and secondly, these MSA consensus sequences were used as queries to search by psi-blast the proteomes used for Hieranoid clustering and in addition a number of monocot species, namely O. sativa (Osa), Zea mays (Zma), Triticum urartu (Tur), Sorghum bicolor (Sbi), Hordeum vulgare (Hvu), Brachypodium distachyon (Bdi), and Aegilops tauschii (Ata) (group B) (Figure 2, P3; see P3_psiblastProcedure.txt in Additional file 3: File S2).

The E-values were then compared between the groups by Wilcoxon test (Figure 2, P4; see P4_eval_wilcox.R in Additional file 3: File S2) to identify genes for which 


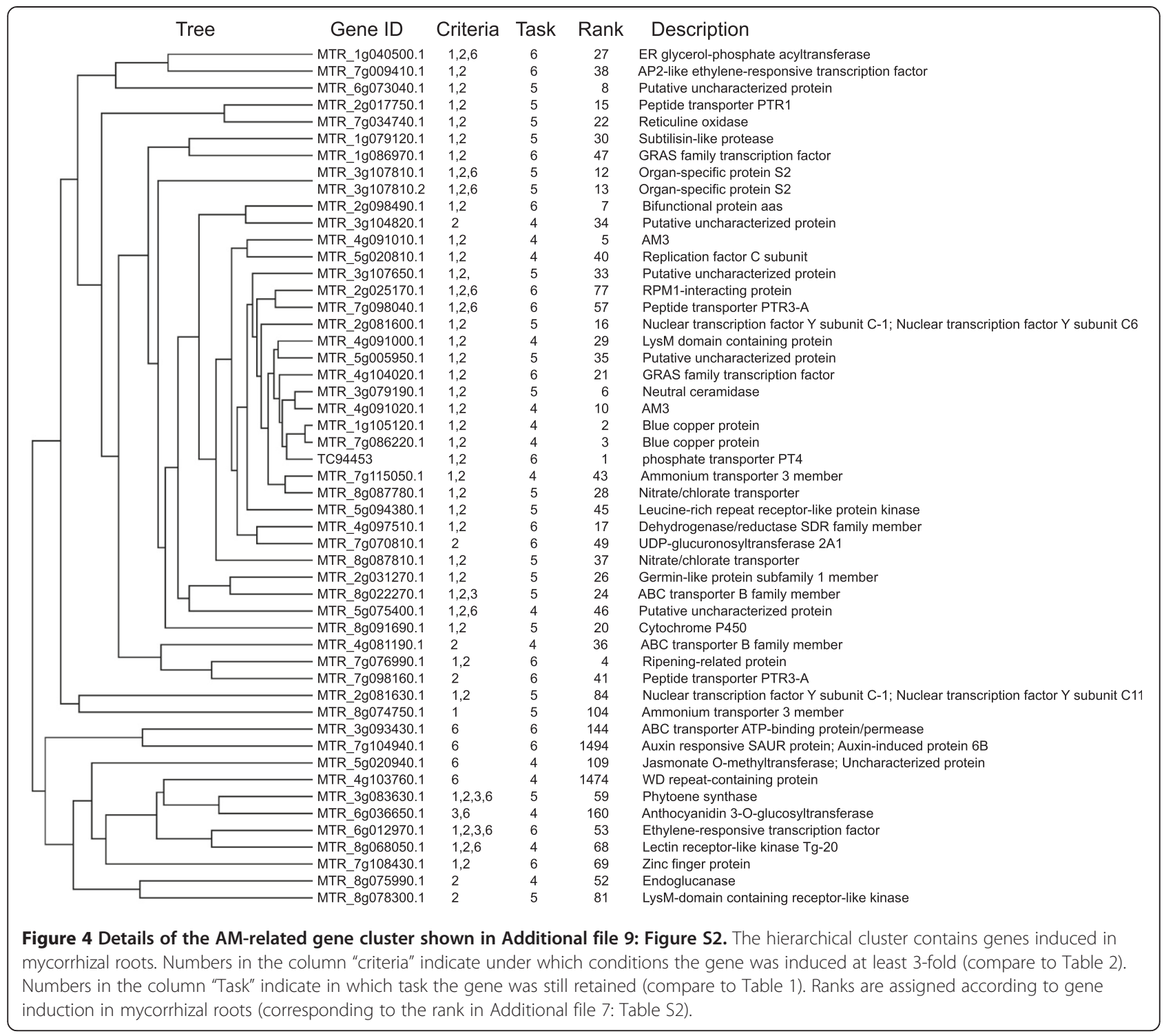

the populations of E-values between groups $\mathrm{C}$ and $\mathrm{A}$, or between $\mathrm{C}$ and $\mathrm{B}$ were significantly different (Additional file 12: Table S4). For proteins that exhibited significant differences, the E-values were averaged among the three groups, and the ratios between the $\log (10)$ of these values for $\mathrm{C} / \mathrm{A}$ and $\mathrm{C} / \mathrm{B}$ were calculated as a relative measure for AM-related sequence conservation (conservation ratio). The higher the conservation ratio, the farther the nonmycorrhizal homologues are from the consensus sequences relative to the homologues from AM-competent

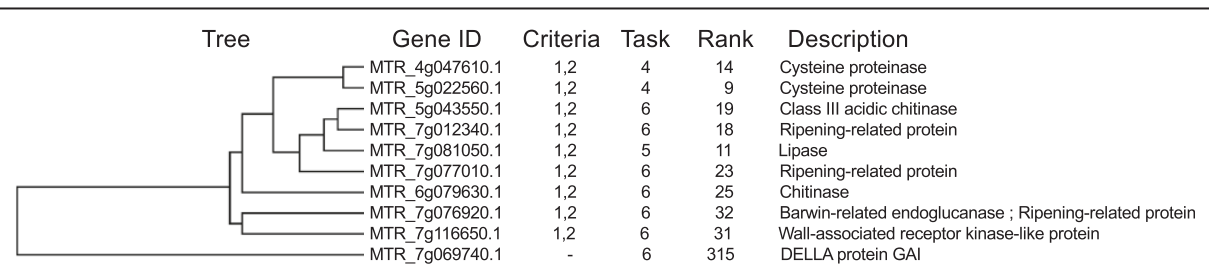

Figure 5 Cluster of genes induced in both, mycorrhizal roots, and nodule symbiosis. Details from cluster shown in Additional file 8: Table S3. Numbers in the column "criteria" indicate under which conditions the gene was induced at least 3-fold (compare to Table 2). Numbers in the column "Task" indicate in which task the gene was still retained (compare to Table 1). Ranks are assigned according to gene induction in mycorrhizal roots (corresponding to the rank in Additional file 7: Table S2). 
species, indicative for AM-related conservation. Establishing the frequency distribution of the conservation ratios revealed that several of our test genes, such as SYMRK, VAPYRIN, RAM1, RAM2, and PT4 passed this filter (Figure 6a), hence their pattern of sequence conservation was significantly related to the competence to engage in AM symbiosis. Surprisingly, none of the test genes passed the comparison between non-mycorrhizal dicots and mycorrhizal monocots (Figure 6b), although at least VAPYRIN, RAM1, and PT4 are more closely related between the AM-competent dicots and the monocots, than between the AM-competent and the non-mycorrhizal dicots [16] (Figure 1a,b).

Since the significance threshold of the Wilcoxon test eliminated many genes, we sought for an alternative way to evaluate the degree of AM-related conservation. Instead of a fixed threshold level, we defined the conservation ratios for a set of household proteins that do not exhibit an AM-related bias in conservation. To this end, we extracted from the Hieranoid clusters those that have representative homologues from all plants, including AM-competent and non-mycorrhizal species (results from Task9; Additional file 13: Table S5). Thus, this set contains conserved house-keeping proteins that can serve as reference for the conservation patterns of proteins identified by Task3. We proceeded in the same way as with the proteins identified by Task3, i.e. an MSA consensus sequence was calculated based on the 6 AM-competent species from each protein cluster, and these MSA sequences were used as queries for psi-blast against all proteomes. As expected, most of the genes selected in this way showed a conservation pattern consistent with the closer phylogenetic relatedness among all the dicots vs. the monocots (Additional file 13: Table S5). This fact is reflected in the phylogenetic trees of the housekeeping genes cyclin D6 and RPL5 (Figure 1a and b), which are also represented in the list resulting from Task9 (Additional file 13: Table S5).

We selected a set of 150 proteins from the results of Task9 with intermediate E-values (excluding E-value =0) and with genes represented in most monocots (excluding genes marked with $\mathrm{NaN}$ in Additional file 13: Table S5). For these 150 genes (marked in yellow in Additional file 14: Table S6), the ratios were calculated as for the genes obtained with Task3 (see above). These values tended to be more to the negative, reflecting the closer position of the Brassicaceae homologues from the MSA consensus sequences relative to the proteins obtained with Task4. Hence, the 150 reference genes resulting from Task9 define the range of conservation ratios for housekeeping proteins and therefore allows to define the range that contains proteins with an AM-related bias of conservation (Additional file 14: Table S6).

Global comparison of the conservation ratios of proteins identified by Task 3 and Task9, revealed considerably higher values for the former group (Figure 7), reflecting the different conservation patterns among proteins selected by Task3 and Task9. In the comparison between group A (AM-competent dicots) and group C (non-mycorrhizal dicots), the AM-related genes RAM1 and PT4 were clearly (a)

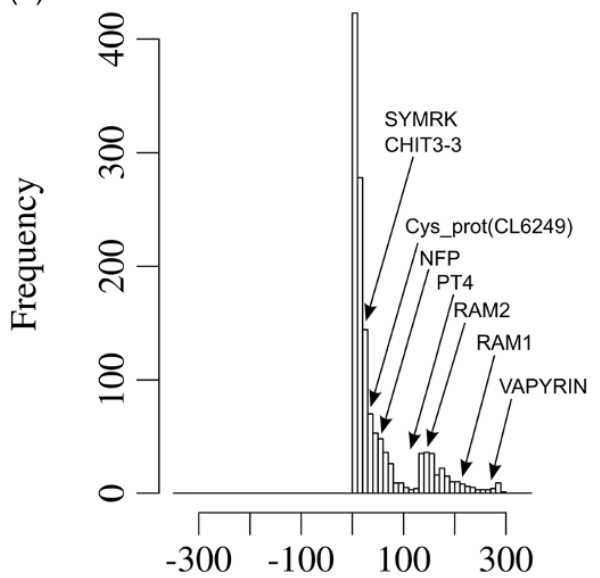

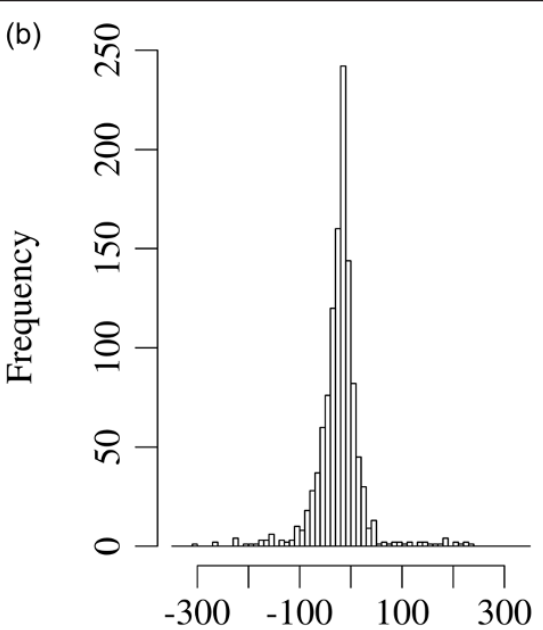

Figure 6 Conservation ratios of potentially AM-related proteins averaged for relevant plant groups with significant difference between non-AM and AM species. Histograms represent the frequency distributions of the ratios of log 10 of the E-values from psi-blast. The query sequences for psi-blast were generated by calculating MSA consensus sequences based on the results of Task3. These query sequences were blasted against AM-competent dicot species (group A), monocot species (group B), and non-mycorrhizal dicot species (group C) (compare with Additional file 12: Table S4). To derive conservation ratios, the E-values were averaged group-wise for groups A, B, and C, respectively, and the following ratios were generated: $C / A$ and $C / B$. Conservation ratios were included only if the difference between the groups were significant ( $p<0.05$ for Wilcoxon test, compare with Additional file 12: Table S4). (a) Ratios for $\log 10$ (group C)/log10(group A). (b) Ratios for $\log 10$ (group C)/log 10 (group B). Note that 8 control genes from Additional file 5: Table S1 passed the filter in (a), whereas none passed in (b). 

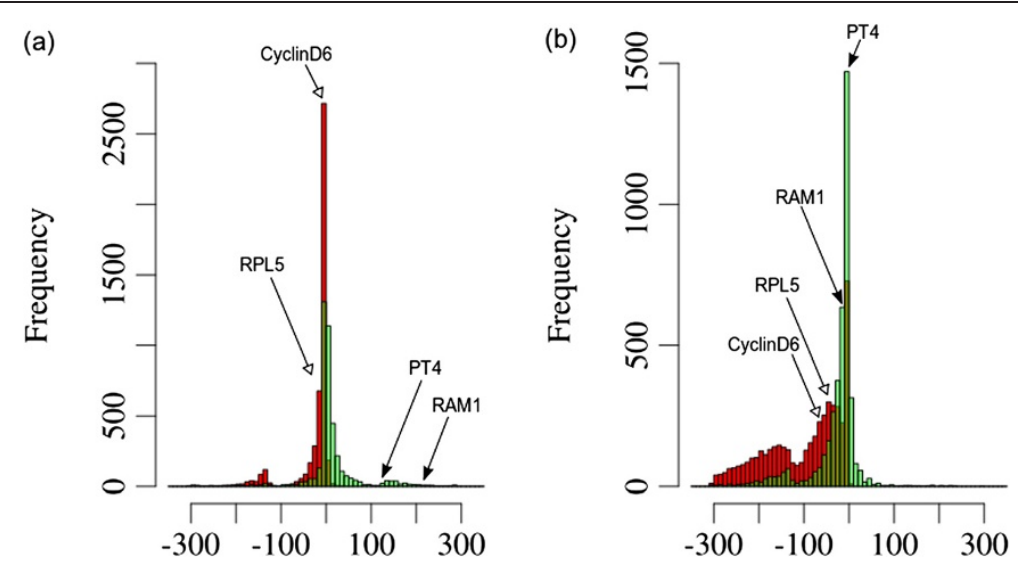

Figure 7 Conservation ratios of potentially AM-related proteins in comparison with housekeeping genes averaged for relevant groups of plant species. Conservation ratios were generated as for Figure 6 (see legend of Figure 6). However, no statistics were performed and all ratios are shown. Conservation ratios are compared for potential AM-related proteins extracted by Task3 (green), and for potential house-keeping genes identified by Task9 (red). For comparison, the position of the proteins represented in Figure 1 is indicated (RAM1: AES78316; PT4: AAM76743; cyclin D6: AES67335; RPL5: AES80278). (a) Ratios for log10(group C)/log10(group A). (b) Ratios for $\log 10$ (group C)/log10(group B).

seprated from the housekeeping controls cyclin D6 and RPL5 (Figure 7a), while this distinction was much less clear in the comparison between group B (AM-competent monocots) and group $\mathrm{C}$ (Figure $7 \mathrm{~b}$ ). These results show that the conservation ratio can be used as a comparative proxy to evaluate the relative degree of conservation of a given protein among AM-competent species relative to the non-mycorrhizal species.

\section{Outcome of the in silico substraction approach}

The goal of this study was to identify AM-related genes based on the conservation pattern of their orthologues between AM-competent and non-mycorrhizal plant species. This approach is particularly targeted to identify AM-related genes that are not induced during symbiosis, hence, we focused on the proteins identified by Task3 that are not induced at the gene level (Additional file 14: Table S6). To evaluate the efficiency of the approach, this list was ordered according to the ratios of E-values between the averaged Brassicaceae and AM-competent dicot plants, respectively (Additional file 14: Table S6). The list was sorted in descending order, since the highest values for the conservation ratios indicate the proteins that are conserved to a higher degree among AM-competent species than between AM-competent and non-mycorrhizal species. In this list, the first protein, a predicted $\alpha$-glucosidase/ xylosidase, was chosen to evaluate its conservation pattern in detail. Indeed, a phylogenetic tree prepared as in Figure 1 shows an extremely skewed pattern of conservation with a clearly resolved common branch of the AM-competent monocots and dicots, including the basal lineage Amborella trichopoda, whereas all the non-mycorrhizal species, the Brassicaceae, and B. vulgaris as a representative of the Chenopodiaceae, form an outlier group (Figure 8a).
Hydrolases are often encoded by gene families, and this is also the case for this $\alpha$-glucosidase/xylosidase. In order to test whether the member with the AM-related conservation pattern forms a dedicated group in AM-competent species, we isolated all the homologues available from the protein database at NCBI for the species $V$. vinifera, $P$. trichocarpa, S. lycopersicum, $M$. truncatula and $A$. thaliana. They are numbered in each species based on their similarity to the AM-related homologue in S. lycopersicum. A phylogenetic tree with all these sequences revealed that all AM-competent species have a single AM-related homologue, resulting in a clearly separated AM-related branch (Figure 8b). The closest homologue of A.thaliana falls into the large containing the remaining sequences. Hence, A. thaliana misses only the AM-related form of the $\alpha$-glucosidase/ xylosidase gene family.

\section{Search for potential cis-regulatory elements in promoters of AM-related genes}

Besides the conservation of the ORF, we investigated the non-coding upstream sequences of AM-related genes by searching for potential cis-regulatory promoter elements that may control gene activity during symbiosis. We selected the $M$. truncatula proteins identified by Task4 that were at least 3-fold induced in mycorrhizal roots relative to non-mycorrhizal control roots (Figure 3, Additional file 7: Table S2 with criteria LCM or AM >3; 190 genes). Their promoter sequences (2 kb upstream of the start codon), were downloaded from Ensembl Plants BioMart (http://plants.ensembl.org/biomart/martview), and analyzed with the pattern recognition software MEME (http://meme.nbcr.net/meme/doc/cite.html) for overrepresented sequences. A first search revealed a series of conserved predicted promoter elements (Additional file 15: 


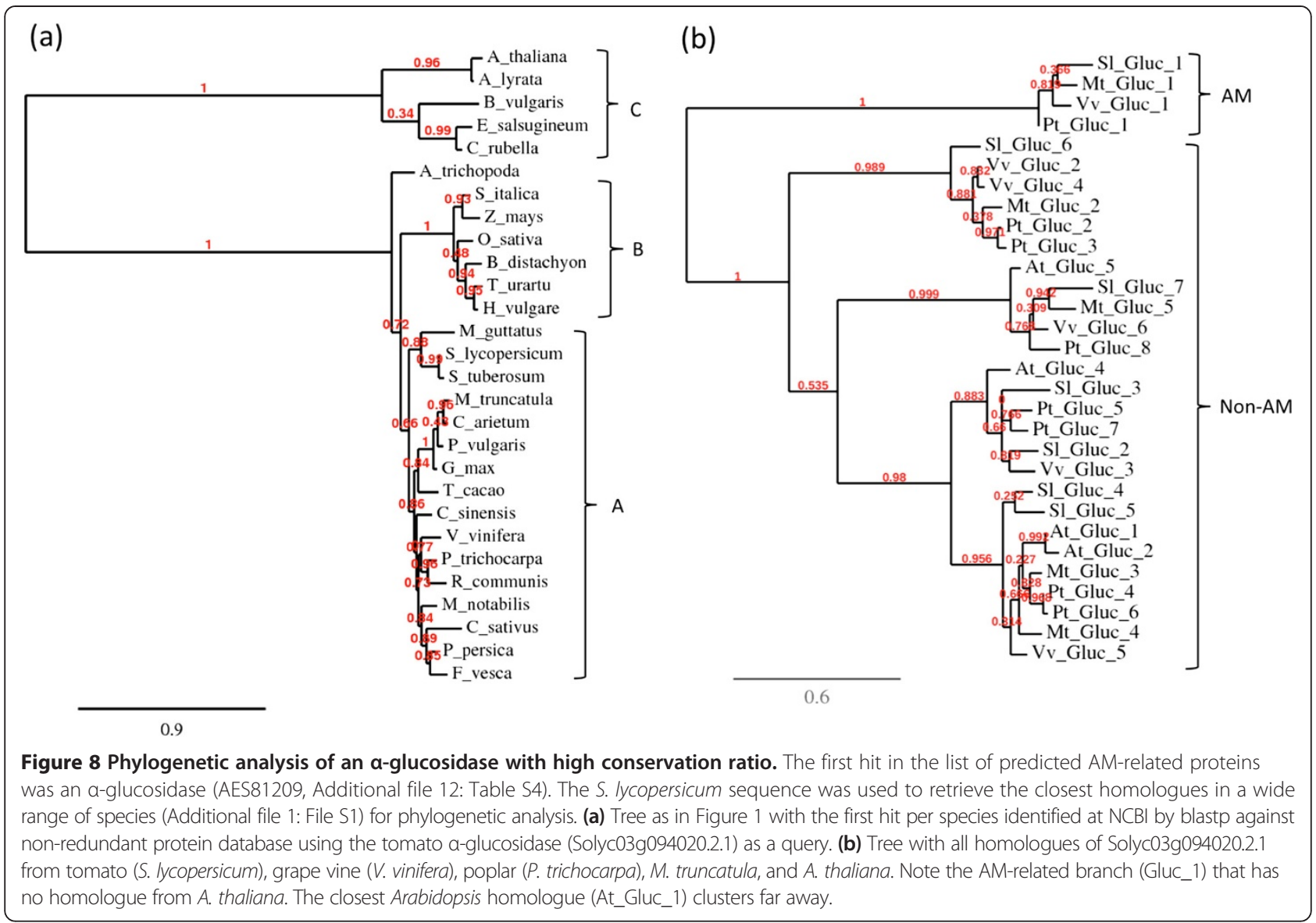

Figure S4a). A highly conserved element comprised the core sequence GGGGTTCGAACCCC (Myc1 element) with the bold letters being almost invariant (Additional file 15: Figure S4, Additional file 16: Table S7). The palindromic nature of this element, and the fact that many cis-elements in plant promoters are palindromes [43-45] prompted us to search specifically for palindromic sequences. Interestingly, 7 of the 10 elements predicted by the first search were confirmed by the second search for palindromes (Additional file 15: Figure S4b, compare with Additional file 15: Figure S4a). Besides the Myc1 element, a second element with the well-defined core sequence TGAGCTTAGCTCA (element Myc2) emerged (Additional file 15: Figure S4). An element with completely invariant sequence was the palindrome GCCGGC that tended to be located within the $500 \mathrm{bp}$ immediately upstream of the ATG (Additional file 17: File S5), indicating that it represents a relevant promoter element (Additional file 17: File S5). Besides the GCCGGC element, none of the predicted elements were described previously. An element that could have been expected to result from our MEME search is the AM-related CTTC element (sequence: CTTGTTC), also known as MYCS element $[46,47]$. This element is present in the promoters of AM-related phosphate transporter genes, and of the AM-related SNARE LjVTI12 [46,47], however, it was not found by MEME in our sample of 190 AM-induced promoter sequences. This surprising result prompted us to search for it in the VAPYRIN promoter, which is induced in mycorrhizal roots of both, M. truncatula and Petunia hybrida $[17,19]$, indicating that this promoter may contain conserved AM-related promoter elements. Indeed, we found an extended version of the CTTC element (sequence GACTTGTTC) in all promoters of 14 VAPYRIN genes from various monocots and dicots, indicating that it is of a wider significance for AM-related gene regulation (data not shown).

The elements Myc1 and Myc2, the GCCGGC element, and the CTTC element were selected for further statistical analysis in the genome of $M$. truncatula. In order to estimate their relative frequency in the promoters of AM-related genes, we selected three samples: the promoters of the genes identified with Task4 $(\mathrm{n}=1547)$, those of the AM-induced genes whithin Task4 $(\mathrm{n}=190)$, and all promoters of $M$. truncatula $(\mathrm{n}=46014)$. These promoter sequences were searched by blast for the presence of the four elements of interest. For the non-palindromic CTTC element, both orientations were considered separately, 
and the two respective longer variants (GACTTGTTC and its reverse complement) were included as well (Additional file 16: Table S7). Indeed, element Myc1 and Myc2, as well as the GCCGGC element were significantly overrepresented among the genes identified by Task 4 compared to the statistical expectations, and relative to the observed frequency in all promoters (Additional file 16: Table S7). Overrepresentation was even more evident when the AM-induced genes were considered (Additional file 16: Table S7). Significance of these results was confirmed by binomial test. In contrast to the Myc1 and Myc2 elements, and to the GCCGGC element, the CTTC element was not overrepresented in promoters identified by Task4, and only moderately in the AM-induced genes, when its original sequence was considered (CTTGTTC) (Additional file 16: Table S7). However, the longer form (GACTTGTTC) was significantly overrepresented in both orientations in the promoters of the genes identified by Task4 and even more of the AM-induced genes (Additional file 16: Table S7). This indicates that the longer version is the relevant form of the CTTC element.

\section{Discussion}

During the past decade, many screens for AM- or RNS-defective mutants have been carried out and a significant number of genes involved in signalling and symbiotic functioning have been identified [4]. In addition, transcript profiling has identified many AM- and RNS-related transcripts, however, only few of them have been functionally characterized by reverse genetic approaches. A central finding of the majority of these genetic studies was, that the genes involved in symbiosis are well conserved among AM-competent species, whereas they are conserved to a lesser extent, or entirely missing, in non-mycorrhizal species such as $A$. thaliana. Based on this finding, we designed a strategy for a third avenue to identify yet unexplored AM-related genes using the conservation of their coding region as the main criterion. We compared the proteomes of AM-competent and non-mycorrhizal plant species, thereby isolating the proteins that are conserved only, or to a higher degree, in AM-competent species. Essentially, this approach represents an in silico substraction procedure, where the proteomes of the non-mycorrhizal species are substracted from the proteomes of the AM-competent species to yield a fraction of the proteome that is expected to be enriched in AM-related proteins.

Since function is selected for at the level of the protein, and since sequence conservation at the DNA level is affected by the degeneracy of the genetic code, we compared the species at the proteome level. Ideally, an in silico substractive approach would involve an equal number of proteomes of the AM-competent and the non-mycorrhizal group. However, due to the wide success of AM in nature, non-mycorrhizal species are the exception (estimated $10-20 \%$ of the angiosperm species), and only few of them have been sequenced to a degree that they could be used for such an approach. As non-mycorrhizal species, only $A$. thaliana, A. lyrata, and B. rapa were available as proteomes in the ENSEMBL database. In contrast, a wide range of AM-competent species is available, among them various dicots (legumes, Solanaceae, etc.) and cereals. Hence, in a first approach, we selected two AM-competent monocots (O. sativa, S. bicolor), four AM-competent dicots (S. lycopersicum, $V$. vinifera, $M$. truncatula, and G. $\max$ ), and the two non-mycorrhizal species $A$. thaliana and $B$. rapa.

The first step in this approach was to establish the orthologous relationships between the proteomes of the eight selected species. This task was performed with Hieranoid, which establishes clusters of orthologous/ paralogous proteins in a hierarchical step-wise procedure that proceeds according to a previously defined phylogenetic tree of the involved species. The subsequent step is to identify protein clusters that have members in all or most of the AM-competent species, but none in the non-mycorrhizal species. However, after a first run with Hieranoid, we noticed that in many cases, the orthologous trees of known single proteins fell into a monocot and a dicot tree, thus preventing our strategy. Therefore, we decided to perform a clustering just using dicot species, namely G. max, M. truncatula, P. trichocarpa, $V$. vinifera, S. lycopersicum, and S. tuberosum, together with the three non-mycorrhizal species $A$. thaliana, A. lyrata, and B. rapa (see Additional file 2: Figure S1). In order to assess the efficiency of the procedure, we used a number of known AM-related genes and a few housekeeping genes for reference (Additional file 5: Table S1).

Clustering of all nine proteomes resulted in a total of 28 '528 protein clusters. Subsequent selection of clusters that contain orthologues only in AM-competent species established a first set of potential AM-related proteins (Figure 2, Task6). In order to account for cases were individual orthologues may be missing from one or two proteomes, we relaxed the stringency of the filtering by allowing also for clusters were 1 or 2 members of AM-competent species were missing (Task 5 and Task4, respectively) (Table 1, Figure 2). An even more permissive filter (Task3) was applied to obtain a large population of proteins that were subsequently subjected to quantitative assessment of amino acid sequence conservation based on the comparison of E-values (Figure 2, Additional file 12: Table S4, Additional file 13: S5 and Additional file 14: S6). The fact that Task3 and Task4 yielded rather long lists of candidate proteins ( $>1000$ clusters) was not considered a problem, since subsequent filtering and sorting narrowed the list further down (see below). 
A next step was to use gene expression as a criterion for selection, hence, we defined expression ratios relevant for AM (Table 2). Based on these criteria we sorted the list of proteins identified by Task4. By applying a stringent threshold induction ratio of 3 -fold, we restricted the analysis to genes with a robust transcriptional induction in mycorrhizal roots (Figure 3, Additional file 7: Table S2). Besides known AM-related proteins such as PT4, AM3, VAPYRIN, RAM2, and IPD3, this list included several interesting proteins like the LysM receptor kinase LYR1, a close homologue of the Lotus japonicus nod factor receptor NFR5 [48], and several ABC transporters and peptide transporters, that may play a role in AM development. Highly induced candidates with yet unknown functions in AM include a triacylglycerol lipase (MTR_7g081050.1), neutral ceramidase (MTR_3g079190.1), an enzyme with a role in sphingolipid metabolism [49], and reticuline oxidase (MTR_7g034740.1) an enzyme involved in the biosynthesis of the alkaloid intermediate reticuline [50]. The fact that many known AM-induced genes were recovered by our selection procedure (Additional file 7 : Table S2 and S3) documents the potential of gene discovery by comparative phylogenetics.

The main goal of this study was to identify new AM-related proteins based on conservation of the coding sequence, i.e. independently of AM-related gene induction. In order to obtain a quantitative measure for conservation of the open reading frame (ORF) in AM-competent species, we performed a secondary filtering step by blasting the conserved consensus sequences derived from the 6 AM-competent species (MSA) against a wider panel of plant proteomes including dicots, monocots, the liverwort Selaginella moellendorfii, the moss Physcomitrella patens, and the unicellular alga Chlamydomonas reinhardtii. The interest to include these three lower plants is based on the finding that several of the AM-related genes were found in liverworths and/or mosses [5,6], potentially revealing the evolutionary origin of the common SYM pathway in lower land plants [8]. The psiblast was performed with the results of the relatively permissive Task3 (protein clusters containing at least 3 of the 6 AM-competent species). With this approach 1329 proteins were identified for which the E-values between the three relevant groups of plant species were significantly different (Wilcoxon test, $\mathrm{p}<0.05$ ) between the non-mycorrhizal species, and the AM-competent dicots and monocots, respectively (Additional file 12: Table S4, Figure 6), indicating that they are conserved particularly well in AM-competent species. Interestingly, the 50 proteins with the lowest p-values (i.e. highest AMrelated conservation) included 13 proteins with annotations as receptors, protein kinases, phosphatases, disease resistant proteins, or G-protein regulating protein, implying roles in signalling.
In view of the essential role of AM in plant nutrition, a highly conserved ammonium transporter (Additional file 12: Table S4, rank 572), and the oligomineral nutrient transporter Nramp5 (rank 495) are of particular interest. Ammonium is the form in which nitrogen is thought to be transferred from the AM fungus to the plant [51,52], and therefore, ammonium transporters are of particular importance in AM [41]. Transporters for other mineral nutrients such as zinc and copper remain to be identified. Many of the conserved proteins were also found to be well conserved in the liverwort $S$. moelledorfii, and some of them even in the moss $P$. patens, whereas $C$. reinhardtii proved to be phylogenetically much more distant (Additional file 12: Table S4).

In order to obtain a direct quantitative measure for the degree of AM-related conservation, we defined the conservation ratio, which represents the ratio of the averaged E-values from psiblast (as log10) between the non-mycorrhizal species, and the AM-competent species (dicots or monocots, respectively) (Additional file 14: Table S6a). For comparison, the results from Task9 (proteins found in all species) were included as representatives of generally well conserved housekeeping proteins. The larger the conservation ratio, the more a protein sequence is divergent between the non-AM plants and the AM-competent species. Therefore, the proteins identified by Task3 have mostly positive values, whereas the housekeeping proteins (Task9) have neutral or negative values (Figures 6 and 7). Of particular interest were proteins with a conservation ratio $>100$, which comprised for example PT4 and RAM1 (Figure 7a), which are known to show a highly AM-related conservation pattern (Figure 1). A general overview over the conservation ratios (Additional file 14: Table S6a) revealed that for 220 proteins, the conservation ratio could not be calculated because they were entirely missing from the psiblast results in the non-mycorrhizal species $(\mathrm{NaN}$ in Additional file 14: Table S6a). In addition, a further 272 proteins exhibited conservation ratios of $>100$, indicative of a high degree of AM-related conservation. In order to define a range above which the conservation ratios can be considered as being skewed towards the AM-competent species, 150 proteins identified by Task9 (houskeeping genes) were included for reference in the results from Task3 (see Additional file 14: Table S6a; proteins marked in yellow).

Among the 220 proteins that were missing from the non-mycorrhizal species (Additional file 14: Table S6a), 123 have unknown functions. Interestingly, three proteins each are predicted chitinases (TASK3_1488, TASK3_1548, TASK3_1649), and glucanases (TASK3_566, TASK3_581, TASK3_4113), indicating potential roles in cell wall modification or signaling. Three predicted CLE peptides (TASK3_3616, TASK3_3634, TASK3_4020) might be 
involved in AM-related signaling as it was shown for RNS [53]. A known component in symbiotic signaling comprised INTERACTING PROTEIN of DMI3 (IPD3)/ CYCLOPS (TASK3_1003), an interactor of the central symbiosis regulator CCaMK encoded by DMI3 [54-56]. High conservation ratios were also found for VAPYRIN (TASK3_795), which is required both for AM and RNS $[16,17,26]$ and VAPYRIN-like (TASK3_1469).

16 proteins exhibited a value $>100$ for both conservation ratios (Additional file 14: Table S6b), i.e. they are highly conserved among both, AM-competent dicots and monocots, relative to the non-mycorrhizal species. Of particular interest is TASK3_196 which encodes a predicted $\alpha$-glucosidase (Additional file 12: Table S4 and Additional file 14: S6b) which is in some species classified as $\alpha$-xylosidase (e.g. XP_006358190.1 in $S$. tuberosum). While the conserved protein is missing from A. thaliana (Figure 8a), there are related glycosidases that occur in all examined plant species including $A$. thaliana, indicating that the non-mycorrhizal model species misses only the AM-related isoform (Figure $8 \mathrm{~b}$ ). The potential role for $\alpha$-glucosidase or $\alpha$-xylosidase in AM is unknown, but given the important contribution of xylose in the hemicelluloses of the cell wall [57], it may be involved in the modifications of cell walls during AM infection and functioning [20]. Interestingly, a predicted $\alpha$-xylosidase of petunia is among the most strongly repressed genes under conditions of high phosphate, which inhibits AM [19].

Five of the 16 proteins with high conservation ratios $(>100)$ represent receptors and protein kinases, of which three (Task3_354, Task3_238, and Task3_4055) are closely related receptor-like protein kinases (RLKs) (Additional file 14: Table S6b). They are similar to S-locus receptor kinase (SRK) [58], which mediates sporophytic self-incompatibility in the Brassicaceae [59], and to the maize receptor kinase ZmPK1 [60]. Phylogenetic analysis showed that they indeed fall into a large clade of proteins found in all AM-competent species, but with considerably lower conservation in the non-mycorrhizal species (Additional file 11: Figure S5). A conserved C-rich stretch in the amino acid sequence is shared among the three AM-related RLKs, as well as with SRK, and ZmPK1 (Additional file 18: Figure S6), indicating that they may have similar tertiary structures mediated by cysteine bridges. Interestingly, ZmPK1 is expressed in roots and young seedlings, rather then floral tissues [60] suggesting that this family of receptor kinases can have other functions than self-incompatibility. Taken together, the examples of $\alpha$-glucosidae/xylosidase and the RLKs show that our approach can identify proteins with AM-related conservation patterns even if they are members of large families. Furthermore, the case of the three related RLKs highlights the potential of our approach to identify proteins that could potentially escape genetic mutant screens because of functional redundancy.

Transcription is controlled primarily by cis-acting regulatory sequences in the promoters of the genes. Hence, co-regulated genes can be expected to share common regulatory elements in their promoters. Based on this logic, we screened the promoters of 190 AM-induced genes for overrepresented sequences that may represent cis-acting regulatory promoter elements. The prediction program MEME identified two new palindromic elements (elements Myc1 and Myc2) and found the GCCGGC element to be overrepresented in AM-induced promoters as well as in the promoters of genes identified by Task4. Statistical analysis confirmed the overrepresentation of these elements in AM-related promoters (Additional file 16: Table S7). The statistical significance of element Myc1 and Myc2 is emphasized by their rather unusual length (14 nt and $13 \mathrm{nt}$, respectively) compared to other cis-acting elements that are typically less then $10 \mathrm{nt}$ in length [61]. In addition, element Myc1 and Myc2 are often coupled (see Additional file 17: File S5), underscoring their relevance for AM-related gene induction, and suggesting that they may function in conjunction.

The predominant position of the GCCGGC element in a range of $500 \mathrm{bp}$ upstream of the ATG start codon is indicative of functional relevance as well. The GCCGGC element can be bound by the transcription factor RAP.2.11 in ethylene-responsive promoters involved in the potassium starvation response of A.thaliana [62], and it was classified as wound- and pathogen-inducible [63]. GCCGGC represents a palindromic variant of the common GCC element (sequence GCCGCC) that is widely found in the promoters of pathogenesis-related genes and in ethylene-responsive promoters of various plants [64-70]. In fact, GCC elements occur in different variants, which are recognized by specific members of the ETHYLENE RESPONSE FACTOR family of transcription factors [69]. The overrepresentation of the GCCGGC element in AM-related promoters may explain the frequency of predicted stress- and pathogen-related transcripts in the transcriptome of mycorrhizal roots in plant species such as rice, potato, petunia, parsley, M. truncatula, and L. japonicus [19,32-35,71-73].

Surprisingly, the AM-related CTTC/MYCS element (sequence: CTTGTTC) [46,74] was not found by MEME in the 190 AM-induced promoters. Furthermore, it was not overrepresented in the genes resulting from Task4, and only moderately in the AM-induced genes (Additional file 16: Table S7). However, an extended form (GACTTGTTC), was found to be significantly overrepresented, indicating that this may represent the complete AM-inducible element in M. truncatula. An overview over the occurrence of predicted promoter elements in the analyzed promoters is provided in Additional file 19: Table S8. 
Taken together, we have identified two new potentially AM-associated cis-regulatory promoter elements. In addition, we identified the GCCGGC element as a potential AM-related element that may indicate an involvement of ethylene in AM development, and we present a modified (elongated) form of the CTTC element. Future work will address the functional relevance of these elements for AM development and functioning,

\section{Conclusions}

We describe a non-targeted approach to identify new AM-related genes by genome-wide comparative sequence analysis of AM-competent and non-mycorrhizal plant species. The validity of this approach has been confirmed by the finding that it identified many known AM-related genes from the genomes of AM-competent plant species. Following this strategy, we identified many new potentially AM-related genes based on the conservation pattern of the coding sequence, on gene expression pattern, and on predicted regulatory promoter elements. These genes will be further evaluated for their predicted function (pathway), the degree of their conservation, and the copy number, or the number of similar genes in the case of gene families. This process will result in a short list of genes that will be further functionally analyzed by insertional mutagenesis in $P$. hybrida, M. truncatula or L. japonicus using established transposon-related protocols [74-76].

\section{Methods}

\section{Phylogenetic sequence analysis}

Phylogenetic sequence analysis for Figures 1 and 9 was performed using the protein sequences provided in Additional file 1: File S1. To obtain these sequences, the first sequence for each group (marked in bold in Additional file 1: File S1; e.g. M. truncatula RAM1 for the RAM1 group) was used as a query for a protein blast at NCBI against the non-redundant protein database. From the results of this blast, the first hit for each plant species was retrieved. Phylogenetic analysis was performed as described [77] with the basic function ("One-click"). Red numbers on the branches of phylogenetic trees represent indices from an approximate Likelyhood Ratio Test (aLRT) [78]. aLRT vales are equivalent to bootstrap values and indicate well supported branches (close to 1) or weakly supported branches (close to 0$)$.

\section{Databases}

For clustering with Hieranoid, the proteomes of the following species were downloaded from the Ensembl Plants website (www.ensemblgenomes.org; database release 20) [79]: Arabidopsis thaliana (Ath), Arabidopsis lyrata (Aly), Brassica rapa (Bra), Medicago truncatula (Mtr),
Glycine max (Gma), Vitis vinifera (Vvi), Solanum lycopersicum (Sly), Solanum tuberosum (Stu) and Populus trichocarpa (Ptr). The proteome database contains 35387 proteins for Ath, 32668 for Aly, 41026 for Bra, 46020 for Mtr, 73320 for Gma, 29928 for Vvi, 34676 for Sly, 56211 for Stu, and 45779 for Ptr. Three protein sequences (PT4, CASTOR and VAPYRIN), that are involved in mycorrhizal development and functioning, were missing from the proteome of $M$. truncatula, and were therefore added to the fasta Mtr file before clustering and further analysis with MtGEA. For calculation of conservation ratios (see below), the proteomes of the following additional species were downloaded: Oryza sativa (Osa), Zea mays (Zma), Triticum urartu (Tur), Sorghum bicolor (Sbi), Hordeum vulgare (Hvu), Brachypodium distachyon (Bdi), and Aegilops tauschii (Ata).

\section{Orthology/homology inference with Hieranoid}

Clustering of the proteomes was performed with the Hieranoid software [25] that is based on a pairwise hierarchical method guided by a phylogenetic tree (Additional file 2: Figure S1). The relative distances between the branches of this tree are irrelevant for Hieranoid, hence, they were arbitrarily set to 4 . Only the topology of the tree is considered in a heuristic process referred to as hierarchical orthology inference. The resulting clusters of homologues protein sequences were saved in a file called eudicotyledons.OGTree.txt (Additional file 4: File S3, script P0_HieraProcedure). Additional filtering steps were applied to this file using the Newick utilities [80] keeping only clusters that contained sequences from at least $\mathrm{X}$ among the six non-Brassicaceae species, but lacked orthologues of all three Brassicaceae proteomes (Additional file 4: File S3, P1_hieranoid_output_treatment.sh). The respective results are named TaskX.

\section{Gene expression data}

All expression data were downloaded from the Medicago truncatula Gene Expression Atlas (MtGEA version 3; http://mtgea.noble.org/v3) [81,82]. Expression values from the treatments listed in Table 2 were downloaded to calculate expression ratios according to criteria 1-6 (Table 2). These probesets used for this approach covered 1110 protein clusters, i.e. approximately $48 \%$ of all clusters resulting from Task4 (see Table 1). In order to remove unreliable probesets, those with the extension “_x_at" and “_s_at", which signifies probes that are not unique in the genome, or that recognize multiple similar genes, respectively, were eliminated. In addition, probesets that showed incomplete identity with the target sequence were removed if they had a "Genome Identity Factoring All Probes in the Same Probeset" inferior to 70\% (MtGEA, MGAG Gene to Affymetrix GeneChip 

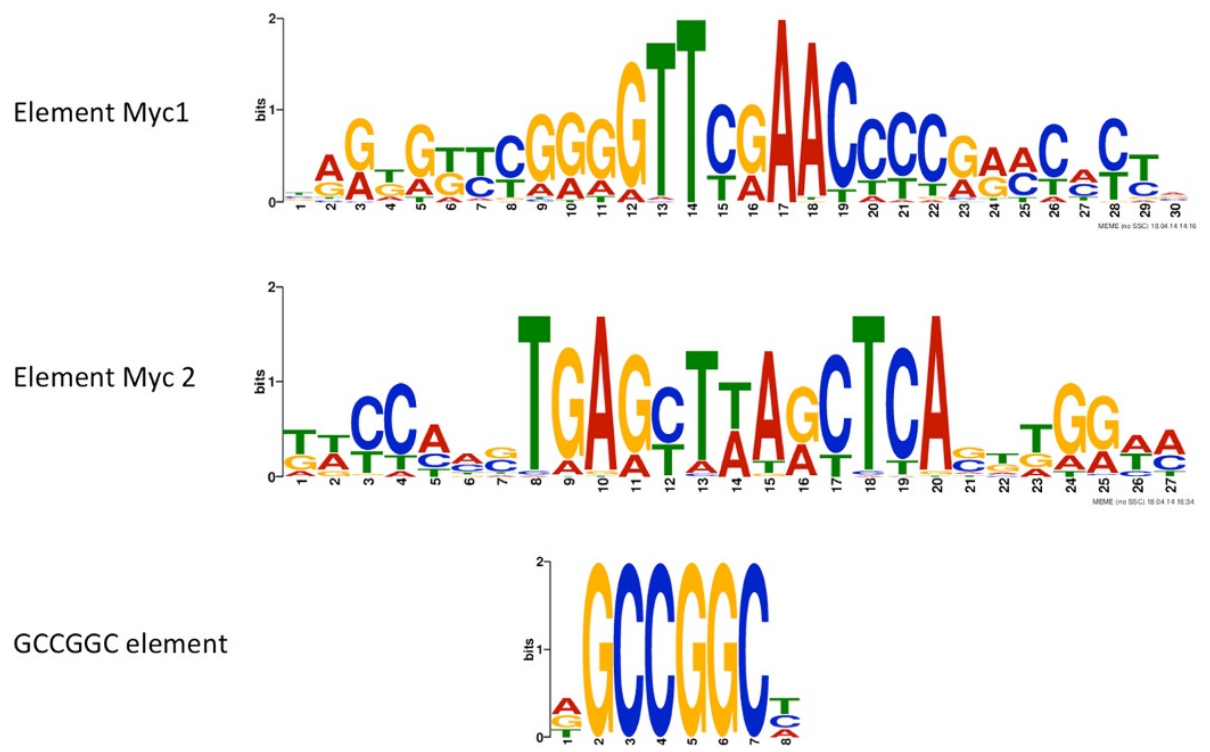

Figure 9 Predicted regulatory elements in the promoters of AM-responsive genes. Promoter elements were identified by MEME from 190 promoters of genes represented in Task4 that are significantly upregulated $(>3 \mathrm{x})$ in mycorrhizal roots or in microdissected cells with arbuscules (red and blue domains in Figure 3, Additional file 8: Table S3). The search was set to palindromic sequences of 6-30 nucleotides.

Mapping). This curation resulted in a set of a total of 1526 Mtr genes that represent 1054 protein clusters (45\% of all clusters identified by Task4).

\section{ClusterMaker}

In order to cluster the genes according to shared expression patterns, the open-source software Cytoscape v. 2.8.3 [83] was used with the ClusterMaker plugin [84] to process the microarray data from MtGEA. The affymetrix values $(\mathrm{x})$ were standardised with the scale-score method as follows (eq. 1).

$$
Y=\frac{(X-\mu)}{\rho}
$$

with $X=\ln (x), \mu$ : average of all $X$ values (from all probesets) per treatment, $\rho$ : standard deviation of all $\mathrm{X}$ values (from all probesets) per treatment. ClusterMaker v.1.11 was run with the following options: "pairwise average-linkage" for Linkage, and "Pearson correlation" for Distance Metric. The option "all the array sources" (Microarray data in log scale) with the choices "Cluster attributes as well as nodes" and "Ignore nodes/edges with no data".

\section{Comparison of conservation level between different plant groups}

First, a consensus sequence was built for each tree in Task3 by multiple sequence alignment (MSA) using MAFFT [42]. These consensus sequences were used as queries to psi-blast the proteomes used for Hieranoid clustering (see above) and an additional set of AMcompetent monocots. Significant differences in the level of amino acid sequence conservation between groups $\mathrm{A}$ (AM-competent dicots), B (AM-competent monocots), and $\mathrm{C}$ (non-mycorrhizal dicots) was determined by Wilcoxon test on the E-values (resulting in the p-values in Additional file 12: Table S4 and Additional file 13: Table S5).

For the calculation of conservation ratios, the E-values were averaged among group A (AM-competent dicots: Mtr, Gma, Sly, Stu, Vvi, Ptr); group B (AM-competent monocots: Osa, Zma, Tur, Sbi, Hvu, Bdi, and Ata); and group C (non-mycorrhizal dicots: Ath, Aly, Bra). Subsequently, the ratios of averaged E-values were calculated to determine the relative relatedness of proteins identified by Task3 (AM-related genes) and Task9 (houskeeping genes) between group $\mathrm{A}, \mathrm{B}$, and $\mathrm{C}$ (Additional file 14: Table S6, Figures 6 and 7).

\section{Analysis of promoter elements}

For the search of potential regulatory elements in promoters, the $2 \mathrm{~kb}$ upstream sequences of all predicted promoters of $M$. truncatula were downloaded from Ensembl Plants BioMart (http://plants.ensembl.org/biomart/martview). To predict potential regulatory cis-acting elements related to $\mathrm{AM}$, the promoters of the genes identified by Task 4 and induced at least 3-fold in AM and/or in cortical cells with arbuscules $(n=190)$ were subjected to analysis by the pattern recognition software MEME (http://meme.nbcr.net/meme/doc/cite.html) [85]. In a first approach, the search was set to sequences of a 
length of 6-30 nt, a second search was performed to identify preferentially palindromic sequences.

To evaluate the frequency of predicted cis-acting elements in the promoters of AM-related genes, the Myc1 and Myc2 element (Figure 9), as well as the GCCGGC element and the CTTC/MYCS element $[46,47]$ were searched for in the promoters of the M. truncatula genes identified by Task $4(\mathrm{n}=1547)$, in the promoters from Task4 that were induced $>3$-fold in mycorrhizal roots and/or in laser-microdissected cells with arbuscules $(\mathrm{n}=190)$, and in all promoters of M. truncatula (46014) by using Jemboss "Nucleic-Motif-Fuzznuc" [86]. Overrepresentation of the predicted elements in the respective samples (relative to the expected frequency based on random sequences) was tested for by binomial test with a coefficient level of 95\% compared to a Fisher value of $a=0.05$ (Additional file 16: Table S7). Fold overrepresentation in promoters from genes identified by Task4 (Task4/all), and in AM-induced promoters (AMup/all) relative to all promoters was calculated as the ratios of the relative frequencies (\% rel. freq.).

\section{Availability of supporting data}

Microarray data are available in the Medicago truncatula Gene Expression Atlas (MtGEA version 3; http://mtgea. noble.org/v3). Plant proteomes were downloaded from the Ensembl Plants website (www.ensemblgenomes.org; database release 20). Promoter sequences of $M$. truncatula were downloaded from Ensembl Plants BioMart (http://plants.ensembl.org/biomart/martview). Phylogenetic trees were prepared with the online tool www.phylogeny.fr using the standard mode ("One-Click") with the sequences provided in Additional file 1: File S1. All Supporting Information has been deposited on LabArchives (http://www.labarchives.com).

\section{Note added in proof}

Since the submission of this manuscript a comparable gene discovery workflow has been published [87]. This paper addresses the evolution of symbiosis-related genes in plants. We provide a comparative list (Additional file 20: Table S9) that documents the overlap of predicted symbiosis-related core genes from Delaux et al. and from our study.

\section{Additional files}

Additional file 1: File S1. Protein sequences used for phylogenetic analysis of RAM1, PT4, RPL5, cyclinD6 (Figure 1), a-glucosidase/xylosidase (Figure 8), and SRK homologues (Additional file 11: Figure S5).

Additional file 2: Figure S1. Conceptual phylogenetic tree of plant species for clustering with Hieranoid. This qualitative phylogenetic tree was used for pair-wise comparison of protein sequences in a step-wise scheme for the construction of protein clusters by Hieranoid. Distances between nodes are irrelevant for this procedure and were set randomly at 4.
Additional file 3: File S2. Collection of procedures and scripts shown in Figure 2. Zip file of procedures (P0 and P3), scripts (P1 and P4) and Cytoscape file (P2) described in Figure 2 and in the Methods. The following scripts (blastcheck.sh, compilMSA.sh, compilMSAinfo.sh, OGT2MSA.pl and task.lua) and data (all9.tre and MappingsMtrAffy.csv) were necessary to properly execute the scripts.

Additional file 4: File S3. Source file from Hieranoid clustering. Text file containing the information for all $28^{\prime} 528$ trees of orthologous/paralogous protein trees used for filtering with Task3 to Task9.

Additional file 5: Table S1. Control proteins as reference for the filtering strategy. Proteins with known function were subjected to filtering by Task4, Task5, and Task6 to test whether the filtering procedure can distinguish AM-related proteins form housekeeping proteins. AM-related proteins were retained either with all three filters $(\leq 6)$, with Task 4 and Task5 $(\leq 5)$, or only through Task 4 ( $\leq 4)$. Proteins were exluded either because they had homologues in non-mycorrhizal species such as A. thaliana (classified as "No"), or because homologues were found in less than 4 AM-competent species (classified as "Not"). See Additional file 6: File $\$ 4$ for cited references.

Additional file 6: File S4. References cited in Additional file 5: Table S1. Additional file 7: Table S2. Expression analysis for genes identified by AM-related conservation of their coding sequence The gene list of Task4 was tested for their expression according to criteria 1-6 (see Table 2). For AM-induced genes (criterion 2), the induction ratios were averaged between the expression results with $R$. irregularis (referred to as $G$. intraradices on the Affymetrix array), and G. mosseae. The list is ordered according to decreasing induction ratios of criterion 2.

Additional file 8: Table S3. Gene lists resulting from combinatorial analysis of gene expression according to criteria 1-6 (compare with Additional file 8: Table S3 and Figure 3). List a: Genes induced only in mycorrhizal roots. List b: Genes induced in mycorrhizal roots and in microdissected roots with arbuscules. List c: Genes induced exclusively in microdissected roots with arbuscules.

Additional file 9: Figure S2. AM-inducible genes identified by clustering based on global gene expression patterns. Hierarchical gene clustering according to gene expression patterns was performed using the entire dataset in MtGEA including all 254 treatments for all probe sets. The cluster was selected based on its high induction of gene expression in mycorrhizal roots (criterion 2). Values corresponding to the different treatments ( $x$-axis) for each gene ( $y$-axis) are displayed in red if the value is above the average scale-score and green if it is lower.

Additional file 10: Figure S3. AM- and nodulation-inducible genes identified by clustering based on global gene expression patterns. Hierarchical gene clustering according to gene expression patterns was performed using the entire dataset in MtGEA including all 254 treatments for all probe sets. The cluster was selected based on its high induction of gene expression in both, mycorrhizal roots (criterion 2) and root nodules. Values corresponding to the different treatments ( $x$-axis) for each gene ( $y$-axis) are displayed in red if the value is above the average scale-score and green if it is lower.

Additional file 11: Figure S5. Phylogenetic tree of receptor-like protein kinases of diverse plant species. An unrooted phylogenetic tree was generated with the three $M$. truncatula sequences identified in Additional file 14: Table S6b (Task3_238, Task3_354, and Task3_4055; see arrows), together with the closest homologues from diverse plant species as indicated. Maize lectine-like protein kinase (ZmPK1; asterisk) is included for reference.

Additional file 12: Table S4. List of AM-related genes resulting from Task3. MSA consensus sequences were computed from Task3 protein clusters and used as queries for psiblast against the proteomes of the indicated species. P-values of $<0.05$ (Wilcoxon-test) indicate significantly different E-values between the groups as indicated.

Additional file 13: Table S5. List of housekeeping genes resulting from Task9. Gene list as in Additional file 12: Table S4, but for proteins identified by Task9 (housekeeping genes).

Additional file 14: Table S6. Proteins with AM-related pattern of sequence conservation. (a) Gene list combined from Additional file 12: Table S4 (AM-related genes) and 150 genes from Additional file 13: Table 
S5 (houskeeping genes). Conservation ratios were calculated by averaging the $\log (10)$ of the E-values per group of plant species $(A, B, C)$, and by dividing $C / A$ and $C / B$. In order to calculate reliable conservation ratios for the housekeeping genes, only the proteins were selected from Additional file 13: Table S5 that had E-values greater than zero, and not more than one missing member in the different plant species. Housekeeping genes are highlighted in yellow to reveal the range of conservation, above which conservation is biased in AM-competent species. The indices TASK3_xxx and TASK9 $x x x$ corresponds to the indices in Additional file 12: Table $S 4$ and Additional file 13: S5, respectively. Protein annotation from different species was obtained by using the first possible hit in the proteomes with the following series of priority: M. truncatula, S. lycopersicum, S. tuberosum, O. sativa A. lyrata, A. thaliana, A. tauschii, B. distachyon, B. rapa, C. reinhardtii, G. max, H. vulgare, P. patens, P. trichocarpa, S. bicolor, S. moellendorfii, T. urartu, Z. mays, and $V$. vinifera. Proteins marked in green represent members with a highly AM-related conservation pattern as shown in Additional file 14: Table S6b. (b) Extract from Additional file 14: Table S6a with proteins with highest conservation ratio (>100) in both, AM-competent dicots and monocots, relative to non-mycorrhizal species.

Additional file 15: Figure S4. Potential cis-regulatory elements predicted from 190 AM-induced promoters. Potential regulatory elements were predicted by MEME for any elements between 6-30 nt (a), and for palindromic elements between 6-30 nt (b). Numbers behind the sequences represent the number of occurrences among the 190 promoters analyzed, and an E-value provided by MEME. Blue lines link related sequences that came up with both searches; black lines underline common sequence elements between related predictions.

Additional file 16: Table S7. Statistical analysis of the frequency of predicted cis-acting elements in the promoters of different groups of genes. The elements predicted by MEME as conserved regulatory cis-elements (Myc1 and Myc2 element, and GCCGGC), and the CTTC element were blasted against the promoters of genes identified by Task4, and of AM-induced genes (AM up). For reference, the predicted promoter elements were blasted against the promoter sequences of all M. truncatula genes. Total: number of promoters (n); Hits: number of promoters with element; $p$-value: Result from binomial test; adjusted p-value: p-value*n; Rel. freq.: Hits/Total.

Additional file 17: File S5. Results from MEME analysis for palindromic regulatory elements in AM-induced promoters. (compare with Figure 9 and Additional file 15: Figure S4).

Additional file 18: Figure S6. Conservation of the C-rich region of S_RLK homologues. Alignement of the C-rich region of Brassica oleracea S_RLK6 (BoSRK6), ZmPK1, and the three M. truncatula proteins identified in this study (Mt238, Mt354, and Mt4055).

Additional file 19: Table S8. Overview of all Medicago truncatula genes corresponding to proteins that showed a significant difference between non-mycorrhizal and AM-competent species. Included are also gene induction in LSM-dissected cortex cells with arbuscules (LCM), and the $\mathrm{p}$-value for the comparison of the E-values from nonAM and AM-competent dicots ( $p$-value Bra/Dic). For the different predicted cis-elements, the number of hits in the respective promoters is listed.

Additional file 20: Table S9. Comparison with the list of conserved genes of Delaux et al. (2014) [87]. The list of 174 conserved AM-related genes (Additional file 20: Table S9 in Delaux et al., 2014 [87]) was

compared with the list of AM-related proteins listed in Additional file 19: Table 58 (see notes added in proof). 50 common genes were identified in both lists. Rank numbers refer to the ranks in Additional file 7: Table S2.

\section{Competing interests}

The authors declare that they have no competing interests.

\section{Authors' contributions}

PF was involved in the design of the bioinformatics strategy, carried out database management and most bioinformatics analysis, and drafted the manuscript. LB was involved in designing the strategy, promoter analysis, and conceiving the manuscript. EB participated in the design of the study and carried out large scale proteome blasts. MD was involved in coordination of the study and carried out statistics. LF conceived the bioinformatics strategy and coordinated the large scale computing efforts. DR was the principle investigator involved in designing the strategy, conceiving the manuscript and coordinating the project. All authors read and approved the final manuscript.

\section{Acknowledgements}

Computations were performed at the Vital-IT (http://www.vital-it.ch) Center for high-performance computing at the Swiss Institute of Bioinformatics (SIB). We wish to thank Mélanie K. Rich for helpful discussions.

\section{Author details}

'Department of Biology, University of Fribourg, Fribourg, Switzerland. ${ }^{2}$ Ludwig Center for Cancer Research, University of Lausanne, Lausanne, Switzerland. ${ }^{3}$ Swiss Institute of Bioinformatics, Fribourg, Switzerland. ${ }^{4}$ Oncology Department, University of Lausanne, Lausanne, Switzerland. ${ }^{5} \mathrm{SIB}$ Swiss Institute of Bioinformatics, Lausanne, Switzerland. ${ }^{6}$ Current address: Crop Genetics, Bayer CropScience NV, Ghent, Belgium.

Received: 17 June 2014 Accepted: 11 November 2014

Published online: 03 December 2014

\section{References}

1. Smith SE, Read DJ: Mycorrhizal Symbiosis. 3rd edition. New York: Academic; 2008.

2. Wang B, Qiu YL: Phylogenetic distribution and evolution of mycorrhizas in land plants. Mycorrhiza 2006, 16(5):299-363.

3. Brundrett MC: Coevolution of roots and mycorrhizas of land plants. New Phytol 2002, 154(2):275-304.

4. Gutjahr C, Parniske M: Cell and developmental biology of arbuscular mycorrhiza symbiosis. Annu Rev Cell Dev Biol 2013, 29:593-617.

5. Delaux PM, Sejalon-Delmas N, Bécard G, Ané JM: Evolution of the plant-microbe symbiotic 'toolkit'. Trends Plant Sci 2013, 18(6):298-304

6. Wang B, Yeun LH, Xue JY, Liu Y, Ane JM, Qiu YL: Presence of three mycorrhizal genes in the common ancestor of land plants suggests a key role of mycorrhizas in the colonization of land by plants. New Phytol 2010, 186(2):514-525.

7. Redecker D, Kodner R, Graham LE: Glomalean fungi from the Ordovician. Science 2000, 289(5486):1920-1921.

8. Kistner C, Parniske M: Evolution of signal transduction in intracellular symbiosis. Trends Plant Sci 2002, 7(11):511-518.

9. Sprent Jl, James EK: Legume evolution: Where do nodules and mycorrhizas fit in? Plant Physio/ 2007, 144(2):575-581.

10. Sprent J: $60 \mathrm{Ma}$ of legume nodulation. What's new? What's changing? J Exp Bot 2008, 59(5):1081-1084.

11. Hocher V, Alloisio N, Auguy F, Fournier P, Doumas P, Pujic P, Gherbi $H$, Queiroux C, Da Silva C, Wincker P, Normand P, Bogusz D: Transcriptomics of actinorhizal symbioses reveals homologs of the whole common symbiotic signaling cascade. Plant Physio/ 2011, 156(2):700-711.

12. Svistoonoff $S$, Benabdoun FM, Nambiar-Veetil M, Imanishi L, Vaissayre $V$, Cesari S, Diagne N, Hocher V, de Billy F, Bonneau J, Wall L, Ykhlef N, Rosenberg C, Bogusz D, Franche C, Gherbi H: The independent acquisition of plant root nitrogen-fixing symbiosis in fabids recruited the same genetic pathway for nodule organogenesis. Plos One 2013, 8(5):e64515. doi:10.1371/journal.pone.0064515.

13. Oldroyd GED: Speak, friend, and enter: signalling systems that promote beneficial symbiotic associations in plants. Nat Rev Microbiol 2013, 11(4):252-263.

14. Oldroyd GED, Downie JA: Nuclear calcium changes at the core of symbiosis signalling. Curr Opin Plant Biol 2006, 9(4):351-357.

15. Singh S, Parniske M: Activation of calcium- and calmodulin-dependent protein kinase (CCaMK), the central regulator of plant root endosymbiosis. Curr Opin Plant Biol 2012, 15(4):444-453.

16. Feddermann N, Duvvuru Muni RR, Zeier T, Stuurman J, Ercolin F, Schorderet $M$, Reinhardt D: The PAM1 gene of petunia, required for intracellular accommodation and morphogenesis of arbuscular mycorrhizal fungi, encodes a homologue of VAPYRIN. Plant J 2010, 64(3):470-481.

17. Pumplin N, Mondo SJ, Topp S, Starker CG, Gantt JS, Harrison MJ: Medicago truncatula Vapyrin is a novel protein required for arbuscular mycorrhizal symbiosis. Plant J 2010, 61(3):482-494. 
18. Feddermann N, Reinhardt D: Conserved residues in the ankyrin domain of VAPYRIN indicate potential protein-protein interaction surfaces. Plant Signaling Behav 2011, 6(5):680-684.

19. Breuillin F, Schramm J, Hajirezaei M, Ahkami A, Favre P, Druege U, Hause B, Bucher M, Kretzschmar T, Bossolini E, Kuhlemeier C, Martinoia E, Franken P, Scholz U, Reinhardt D, Bucher M, Kretzschmar T, Bossolini E, Kuhlemeier C, Martinoia E, Franken P, Scholz U, Reinhardt D: Phosphate systemically inhibits development of arbuscular mycorrhiza in Petunia hybrida and represses genes involved in mycorrhizal functioning. Plant $J$ 2010, 64:1002-1017.

20. Rich MK, Schorderet M, Reinhardt D: The role of the cell wall compartment in mutualistic symbioses of plants. Front Plant Sci 2014, 5:238.

21. Gobbato E, Marsh JF, Vernie T, Wang E, Maillet F, Kim J, Miller JB, Sun J, Bano SA, Ratet P, Mysore KS, Dénarié J, Schultze M, Oldroyd GE: A GRAS-type transcription factor with a specific function in mycorrhizal signaling. Curr Biol 2012, 22(23):2236-2241

22. Zhang Q, Blaylock LA, Harrison MJ: Two Medicago truncatula half-ABC transporters are essential for arbuscule development in arbuscular mycorrhizal symbiosis. Plant Cell 2010, 22(5):1483-1497.

23. Harrison MJ, Dewbre GR, Liu JY: A phosphate transporter from Medicago truncatula involved in the acquisiton of phosphate released by arbuscular mycorrhizal fungi. Plant Cell 2002, 14(10):2413-2429.

24. Javot H, Penmetsa RV, Terzaghi N, Cook DR, Harrison MJ: A Medicago truncatula phosphate transporter indispensable for the arbuscular mycorrhizal symbiosis. Proc Natl Acad Sci U S A 2007, 104(5):1720-1725.

25. Schreiber F, Sonnhammer ELL: Hieranoid: Hierarchical Orthology Inference. J Mol Biol 2013, 425(11):2072-2081.

26. Murray JD, Duvvuru Muni R, Torres-Jerez I, Tang Y, Allen S, Andriankaja M, Li G, Laxmi A, Cheng X, Wen J, Vaughan D, Schultze M, Sun J, Charpentier M, Oldroyd G, Tadege M, Ratet P, Mysore KS, Chen R, Udvardi MK: Vapyrin, a gene essential for intracellular progression of arbuscular mycorrhizal symbiosis, is also essential for infection by rhizobia in the nodule symbiosis of Medicago truncatula. Plant J 2011, 65(2):244-252.

27. Apweiler R, Bateman A, Martin MJ, O'Donovan C, Magrane M, Alam-Faruque Y, Alpi E, Antunes R, Arganiska J, Casanova EB, Bely B, Bingley M, Bonilla C, Britto R, Bursteinas B, Mun Chan W, Chavali G, Cibrian-Uhalte E, Da Silva A, De Giorgi M, Fazzini F, Gane P, Castro LG, Garmiri P, Hatton-Ellis E, Hieta R, Huntley R, Legge D, Liu W, Luo J, et al: Activities at the Universal Protein Resource (UniProt). Nucleic Acids Res 2014, 42(D1):D191-D198.

28. Groth M, Takeda N, Perry J, Uchida H, Draexl S, Brachmann A, Sato S, Tabata S, Kawaguchi M, Wang TL, Parniske M: NENA, a Lotus japonicus homolog of Sec13, is required for rhizodermal infection by arbuscular mycorrhiza fungi and rhizobia but dispensable for cortical endosymbiotic development. Plant Cell 2010, 22:2509-2526.

29. Kanamori N, Madsen LH, Radutoiu S, Frantescu M, Quistgaard EMH, Miwa H, Downie JA, James EK, Felle HH, Haaning LL, Jensen TH, Sato S, Nakamura Y, Tabata S, Sandal N, Stougaard J: A nucleoporin is required for induction of $\mathrm{Ca} 2+$ spiking in legume nodule development and essential for rhizobial and fungal symbiosis. Proc Natl Acad Sci U S A 2006, 103(2):359-364.

30. Saito K, Yoshikawa M, Yano K, Miwa H, Uchida H, Asamizu E, Sato S, Tabata S, Imaizumi-Anraku H, Umehara $Y$, Kouchi H, Murooka Y, Szczyglowski K, Downie JA, Parniske M, Hayashi M, Kawaguchi M: NUCLEOPORIN85 is required for calcium spiking, fungal and bacterial symbioses, and seed production in Lotus japonicus. Plant Cell 2007, 19(2):610-624.

31. Fiorilli V, Catoni M, Miozzi L, Novero M, Accotto GP, Lanfranco L: Global and cell-type gene expression profiles in tomato plants colonized by an arbuscular mycorrhizal fungus. New Phytol 2009, 184(4):975-987.

32. Guether M, Balestrini R, Hannah M, He J, Udvardi M, Bonfante P: Genome-wide reprogramming of regulatory networks, cell wall and membrane biogenesis during arbuscular-mycorrhizal symbiosis in Lotus japonicus. New Phytol 2009, 182(1):200-212

33. Güimil S, Chang HS, Zhu T, Sesma A, Osbourn A, Roux C, Ionnidis V, Oakeley EJ, Docquier M, Descombes P, Briggs SP, Paszkowski U: Comparative transcriptomics of rice reveals an ancient pattern of response to microbial colonization. Proc Natl Acad Sci U S A 2005, 102(22):8066-8070.

34. Hohnjec N, Vieweg ME, Puhler A, Becker A, Küster H: Overlaps in the transcriptional profiles of Medicago truncatula roots inoculated with two different Glomus fungi provide insights into the genetic program activated during arbuscular mycorrhiza. Plant Physiol 2005, 137(4):1283-1301.
35. Liu JY, Blaylock LA, Endre G, Cho J, Town CD, VandenBosch KA, Harrison MJ: Transcript profiling coupled with spatial expression analyses reveals genes involved in distinct developmental stages of an arbuscular mycorrhizal symbiosis. Plant Cell 2003, 15(9):2106-2123.

36. Lévy J, Bres C, Geurts R, Chalhoub B, Kulikova O, Duc G, Journet EP, Ané JM, Lauber E, Bisseling T, Dénarié J, Rosenberg C, Debellé F: A putative Ca2+ and calmodulin-dependent protein kinase required for bacterial and fungal symbioses. Science 2004, 303(5662):1361-1364.

37. Mitra RM, Gleason CA, Edwards A, Hadfield J, Downie JA, Oldroyd GED, Long SR: A Ca2+/calmodulin-dependent protein kinase required for symbiotic nodule development: Gene identification by transcript-based cloning. Proc Natl Acad Sci U S A 2004, 101(13):4701-4705.

38. Nagy F, Karandashov V, Chague W, Kalinkevich K, Tamasloukht M, Xu GH, Jakobsen I, Levy AA, Amrhein N, Bucher M: The characterization of novel mycorrhiza-specific phosphate transporters from Lycopersicon esculentum and Solanum tuberosum uncovers functional redundancy in symbiotic phosphate transport in solanaceous species. Plant J 2005, 42(2):236-250

39. Rausch C, Daram P, Brunner S, Jansa J, Laloi M, Leggewie G, Amrhein N, Bucher M: A phosphate transporter expressed in arbuscule-containing cells in potato. Nature 2001, 414(6862):462-466.

40. Wegmüller S, Svistoonoff S, Reinhardt D, Stuurman J, Amrhein N, Bucher M: A transgenic dTph1 insertional mutagenesis system for forward genetics in mycorrhizal phosphate transport of Petunia. Plant J 2008, 54(6):1115-1127.

41. Guether M, Neuhauser B, Balestrini R, Dynowski M, Ludewig U, Bonfante P: A mycorrhizal-specific ammonium transporter from Lotus japonicus acquires nitrogen released by arbuscular mycorrhizal fungi. Plant Physiol 2009, 150(1):73-83.

42. Katoh K, Standley DM: MAFFT multiple sequence alignment software version 7: Improvements in performance and usability. Mol Biol Evol 2013, 30(4):772-780

43. Shaikhali J, Noren L, Barajas-Lopez JD, Srivastava V, Konig J, Sauer UH, Wingsle G, Dietz KJ, Strand A: Redox-mediated mechanisms regulate DNA binding activity of the G-group of basic region leucine zipper (bZIP) transcription factors in Arabidopsis. J Biol Chem 2012, 287(33):27510-27525.

44. Vickers CE, Xue GP, Gresshoff PM: A novel cis-acting element, ESP, contributes to high-level endosperm-specific expression in an oat globulin promoter. Plant Mol Biol 2006, 62(1-2):195-214.

45. Zhou P, Yang F, Yu JJ, Ao GM, Zhao Q: Several cis-elements including a palindrome involved in pollen-specific activity of SBgLR promoter. Plant Cell Rep 2010, 29(5):503-511.

46. Chen $A Q$, Gu MA, Sun $S B$, Zhu LL, Hong SA, Xu GH: Identification of two conserved cis-acting elements, MYCS and P1BS, involved in the regulation of mycorrhiza-activated phosphate transporters in eudicot species. New Phytol 2012, 189(4):1157-1169.

47. Lota F, Wegmüller S, Buer B, Sato S, Bräutigam A, Hanf B, Bucher M: The cis-acting CTTC-P1BS module is indicative for gene function of LjVTI12, a Qb-SNARE protein gene that is required for arbuscule formation in Lotus japonicus. Plant J 2013, 74(2):280-293.

48. Radutoiu S, Madsen LH, Madsen EB, Felle HH, Umehara Y, Gronlund M, Sato S, Nakamura Y, Tabata S, Sandal N, Stougaard J: Plant recognition of symbiotic bacteria requires two LysM receptor-like kinases. Nature 2003, 425(6958):585-592

49. Markham JE, Lynch DV, Napier JA, Dunn TM, Cahoon EB: Plant sphingolipids: function follows form. Curr Opin Plant Biol 2013 16(3):350-357.

50. Kutchan TM: Heterologous expression of alkaloid biosynthetic genes A review. Gene 1996, 179(1):73-81.

51. Govindarajulu M, Pfeffer PE, Jin HR, Abubaker J, Douds DD, Allen JW, Bücking H, Lammers PJ, Shachar-Hill Y: Nitrogen transfer in the arbuscular mycorrhizal symbiosis. Nature 2005, 435(7043):819-823.

52. Tian CJ, Kasiborski B, Koul R, Lammers PJ, Bücking H, Shachar-Hill Y: Regulation of the nitrogen transfer pathway in the arbuscular mycorrhizal symbiosis: Gene characterization and the coordination of expression with nitrogen flux. Plant Physiol 2010, 153(3):1175-1187.

53. Miyawaki K, Tabata R, Sawa S: Evolutionarily conserved CLE peptide signaling in plant development, symbiosis, and parasitism. Curr Opin Plant Biol 2013, 16(5):598-606.

54. Chen CY, Ané JM, Zhu HY: OsIPD3, an ortholog of the Medicago truncatula DMI3 interacting protein IPD3, is required for mycorrhizal symbiosis in rice. New Phytol 2008, 180(2):311-315. 
55. Horvath B, Yeun LH, Domonkos A, Halasz G, Gobbato E, Ayaydin F, Miro K, Hirsch S, Sun JH, Tadege M, Ratet P, Mysore KS, Ané JM, Oldroyd GE, Kaló P: Medicago truncatula IPD3 Is a member of the common symbiotic signaling pathway required for rhizobial and mycorrhizal symbioses. $\mathrm{Mol}$ Plant-Microbe Interact 2011, 24(11):1345-1358.

56. Yano K, Yoshida S, Muller J, Singh S, Banba M, Vickers K, Markmann K, White C, Schuller B, Sato S, Asamizu E, Tabata S, Murooka Y, Perry J, Wang TL, Kawaguchi M, Imaizumi-Anraku H, Hayashi M, Parniske M: CYCLOPS, a mediator of symbiotic intracellular accommodation. Proc Natl Acad Sci U S A 2008, 105(51):20540-20545.

57. Scheller HV, Ulvskov P: Hemicelluloses. Annu Rev Plant Biol 2010, 61:263-289.

58. Stein JC, Howlett B, Boyes DC, Nasrallah ME, Nasrallah JB: Molecular cloning of a putative receptor protein kinase gene encoded at the self-incompatibility locus of Brassica oleracea. Proc Natl Acad Sci U S A 1991, 88(19):8816-8820.

59. Nasrallah JB, Nasrallah ME: S-locus receptor kinase signalling. Biochem Soc Trans 2014, 42:313-319.

60. Walker JC, Zhang R: Relationship of a putative receptor protein kinase from maize to the S-locus glycoproteins of Brassica. Nature 1990, 345(6277):743-746.

61. Hernandez-Garcia CM, Finer JJ: Identification and validation of promoters and cis-acting regulatory elements. Plant Sci 2014, 217:109-119.

62. Kim MJ, Ruzicka D, Shin R, Schachtman DP: The Arabidopsis AP2/ERF transcription factor RAP2.11 modulates plant response to low-potassium conditions. Mol Plant 2012, 5(5):1042-1057.

63. Ramkumar G, Madhav MS, Biswal AK, Rama Devi SJS, Sakthivel K, Mohan MK, Umakanth B, Mangrauthia SK, Sundaram RM, Viraktamath BC: Genome-wide identification and characterization of transcription factor binding motifs of NBS-LRR genes in rice and Arabidopsis. J Genomes Exomes 2014, 3:7-15.

64. Cheng MC, Liao PM, Kuo WW, Lin TP: The Arabidopsis ETHYLENE RESPONSE FACTOR1 regulates abiotic stress-responsive gene expression by binding to different cis-acting elements in response to different stress signals. Plant Physiol 2013, 162(3):1566-1582.

65. Hao DY, Yamasaki K, Sarai A, Ohme-Takagi M: Determinants in the sequence specific binding of two plant transcription factors, CBF1 and NtERF2, to the DRE and GCC motifs. Biochemistry 2002, 41(13):4202-4208.

66. Hu FY, Wang D, Zhao XQ, Zhang T, Sun HX, Zhu LH, Zhang F, Li LJ, Li QO, Tao DY, Fu B, Li Z: Identification of rhizome-specific genes by genome-wide differential expression analysis in Oryza longistaminata. BMC Plant Biol 2011, $11: 18$

67. Lu X, Jiang WM, Zhang L, Zhang F, Zhang FY, Shen Q, Wang GF, Tang KX: AaERF1 Positively Regulates the Resistance to Botrytis cinerea in Artemisia annua. Plos One 2013, 8(2):e57657. doi:10.1371/journal. pone.0057657.

68. Pirrello J, Prasad BCN, Zhang WS, Chen KS, Mila I, Zouine M, Latche A, Pech JC, Ohme-Takagi M, Regad F, Bouzayen M: Functional analysis and binding affinity of tomato ethylene response factors provide insight on the molecular bases of plant differential responses to ethylene. BMC Plant Biol 2012, 12:190.

69. Shoji T, Mishima M, Hashimoto T: Divergent DNA-Binding Specificities of a Group of ETHYLENE RESPONSE FACTOR Transcription Factors Involved in Plant Defense. Plant Physiol 2013, 162(2):977-990.

70. Zhou JM, Tang XY, Martin GB: The Pto kinase conferring resistance to tomato bacterial speck disease interacts with proteins that bind a cis-element of pathogenesis-related genes. EMBO J 1997, 16(11):3207-3218.

71. Franken P, Gnädinger F: Analysis of parsley arbuscular endomycorrhiza Infection, development and messenger-RNA levels of defense-related genes. Mol Plant-Microbe Interact 1994, 7(5):612-620.

72. Gallou A, Declerck S, Cranenbrouck S: Transcriptional regulation of defence genes and involvement of the WRKY transcription factor in arbuscular mycorrhizal potato root colonization. Funct Integr Genom 2012, 12(1):183-198.

73. Wulf A, Manthey K, Doll J, Perlick AM, Linke B, Bekel T, Meyer F, Franken P, Kuster $\mathrm{H}$, Krajinski F: Transcriptional changes in response to arbuscular mycorrhiza development in the model plant Medicago truncatula. $\mathrm{Mol}$ Plant-Microbe Interact 2003, 16(4):306-314.

74. Fukai E, Soyano T, Umehara Y, Nakayama S, Hirakawa H, Tabata S, Sato S, Hayashi M: Establishment of a Lotus japonicus gene tagging population using the exon-targeting endogenous retrotransposon LORE1. Plant $J$ 2012, 69(4):720-730
75. Tadege M, Ratet $P$, Mysore KS: Insertional mutagenesis: a Swiss army knife for functional genomics of Medicago truncatula. Trends Plant Sci 2005, 10(5):229-235

76. Vandenbussche $M$, Janssen A, Zethof J, van Orsouw N, Peters J, van Eijk MJT, Rijpkema AS, Schneiders H, Santhanam P, de Been M, van Tunen A, Gerats T: Generation of a 3D indexed Petunia insertion database for reverse genetics. Plant J 2008, 54(6):1105-1114.

77. Dereeper A, Guignon V, Blanc G, Audic S, Buffet S, Chevenet F, Dufayard JF, Guindon S, Lefort V, Lescot M, Claverie JM, Gascuel O: Phylogeny.fr: robust phylogenetic analysis for the non-specialist. Nucleic Acids Res 2008, 36:W465-W469.

78. Anisimova M, Gascuel O: Approximate likelihood-ratio test for branches: A fast, accurate, and powerful alternative. Syst Biol 2006, 55(4):539-552.

79. Kersey PJ, Staines DM, Lawson D, Kulesha E, Derwent P, Humphrey JC, Hughes DST, Keenan S, Kerhornou A, Koscielny G, Langridge N, McDowall MD, Megy K, Maheswari U, Nuhn M, Paulini M, Pedro H, Toneva I, Wilson D, Yates A, Birney E: Ensembl Genomes: an integrative resource for genome-scale data from non-vertebrate species. Nucleic Acids Res 2012, 40(D1):D91-D97.

80. Junier T, Zdobnov EM: The Newick utilities: high-throughput phylogenetic tree processing in the Unix shell. Bioinformatics 2010, 26(13):1669-1670.

81. Benedito VA, Torres-Jerez I, Murray JD, Andriankaja A, Allen S, Kakar K, Wandrey M, Verdier J, Zuber H, Ott T, Moreau S, Niebel A, Frickey T, Weiller G, He J, Dai X, Zhao P, Tang Y, Udvardi M, Moreau S, Niebel A, Frickey T, Weiller G, He J, Dai X, Zhao P, Tang Y, Udvardi M: A gene expression atlas of the model legume Medicago truncatula. Plant J 2008, 55(3):504-513.

82. He J, Benedito VA, Wang MY, Murray JD, Zhao PX, Tang YH, Udvardi MK: The Medicago truncatula gene expression atlas web server. BMC Bioinformatics 2009, 10:441.

83. Smoot ME, Ono K, Ruscheinski J, Wang PL, Ideker T: Cytoscape 2.8: new features for data integration and network visualization. Bioinformatics 2011, 27(3):431-432.

84. Morris JH, Apeltsin L, Newman AM, Baumbach J, Wittkop T, Su G, Bader GD, Ferrin TE: clusterMaker: a multi-algorithm clustering plugin for Cytoscape. BMC Bioinformatics 2011, 12:436.

85. Bailey TL, Boden M, Buske FA, Frith M, Grant CE, Clementi L, Ren JY, Li WW, Noble WS: MEME SUITE: tools for motif discovery and searching. Nucleic Acids Res 2009, 37:W202-W208.

86. Rice $\mathrm{P}$, Longden I, Bleasby A: EMBOSS: The European molecular biology open software suite. Trends Genet 2000, 16(6):276-277.

87. Delaux PM, Varala K, Edger PP, Coruzzi GM, Pires JC, Ané JM: Comparative phylogenomics uncovers the impact of symbiotic associations on host genome evolution. PLOS Genet 2014, 10(7):e1004487

doi:10.1186/s12870-014-0333-0

Cite this article as: Favre et al:: A novel bioinformatics pipeline to discover genes related to arbuscular mycorrhizal symbiosis based on their evolutionary conservation pattern among higher plants. BMC Plant Biology 2014 14:333.

\section{Submit your next manuscript to BioMed Central and take full advantage of:}

- Convenient online submission

- Thorough peer review

- No space constraints or color figure charges

- Immediate publication on acceptance

- Inclusion in PubMed, CAS, Scopus and Google Scholar

- Research which is freely available for redistribution 